\title{
Ferroptosis: A Potential Target in Cardiovascular Disease
}

\author{
Yanlong Leng ${ }^{1,2 \dagger}$, Xing Luo ${ }^{1,2 \dagger}$, Jiaying $\mathrm{Yu}^{2,3}$, Haibo Jia ${ }^{1,2 *}$ and Bo $\mathrm{Yu}^{1,2}$ \\ ${ }^{1}$ Department of Cardiology, The Second Affiliated Hospital of Harbin Medical University, Harbin, China, ${ }^{2}$ Key Laboratory of \\ Myocardial Ischemia, Harbin Medical University, Harbin, China, ${ }^{3}$ Department of Obstetrics and Gynecology, The Second Affiliated \\ Hospital of Harbin Medical University, Harbin, China
}

\section{OPEN ACCESS}

Edited by:

Chunying $\mathrm{Li}$,

Fourth Military Medical University,

China

Reviewed by:

Takashi Matsui,

University of Hawaii, United States

Weinan Guo,

Fourth Military Medical University,

China

Xin Wei,

Second Hospital of Anhui Medical

University, China

${ }^{*}$ Correspondence:

Haibo Jia

jhb101180@163.com

tThese authors have contributed equally to this work and share first

authorship

Specialty section:

This article was submitted to

Cell Death and Survival,

a section of the journal

Frontiers in Cell and Developmental

Biology

Received: 12 November 2021

Accepted: 31 December 2021

Published: 20 January 2022

Citation:

Leng Y, Luo X, Yu J, Jia $H$ and Yu B (2022) Ferroptosis: A Potential Target

in Cardiovascular Disease.

Front. Cell Dev. Biol. 9:813668.

doi: $10.3389 /$ fcell.2021.813668
Ferroptosis is a new form of regulatory cell death characterized by iron-dependent and intracellular lipid peroxidation. Ferroptosis can be divided into two stages. The first stage is iron overload in the cell, which generates a large amount of reactive oxygen species through the Fenton reaction, and the second stage results from an imbalance of the intracellular antioxidant system. Excessive phospholipid hydroperoxides cannot be removed by reduction reactions, as this could destroy the cell membrane structure and interfere with mitochondrial function, eventually leading to ferroptosis of the cell. Cardiovascular diseases have gradually become the leading cause of death in modern society. The relationship between ferroptosis and the occurrence and progression of cardiovascular disease has become a research hotspot in recent years. In this review, we summarize the mechanism of ferroptosis and its specific role in cardiovascular disease.

Keywords: ferroptosis, cardiovascular disease, iron homeostasis, antioxidant metabolism, lipid metabolism, ischemia reperfusion, heart failure, coronary atherosclerotic heart disease

\section{INTRODUCTION}

Cell death is an important cornerstone for maintaining the balance of development and homeostasis of the body. There are two main types of cell death: regulatory cell death (RCD) and non-regulatory cell death (Lockshin and Zakeri, 2001). Nonregulatory cell death refers mainly to the severe damage of cells under the stimulation of various pathogenic factors, leading to passive cell death. However, according to the different signaling pathways involved in the process, regulatory cell death can be divided into apoptosis, necroptosis, pyroptosis, autophagy, and ferroptosis (Del Re et al., 2019). Among them, ferroptosis is a new type of regulatory cell death discovered only recently. In fact, in 2003, Dolma et al. (Dolma et al., 2003) identified a new type of compound that caused tumor cell death and named it erastin. However, due to limitations in research on regulatory cell death at that time, the authors failed to identify the special regulatory cell death mode induced by erastin. Until 2012, when Dixon et al. defined erastininduced cell death as iron-dependent regulatory cell death induced by lipid peroxidation and named it ferroptosis (Dixon et al., 2012). The morphological characteristics of ferroptosis cells are as follows: mitochondrial shrinkage, decreased mitochondrial cristae, and decreased membrane density, which are different from the characteristic apoptotic body formation of apoptotic cells.

Cardiovascular disease is one of the most threatening diseases to human health today. Cardiovascular diseases mainly include coronary atherosclerotic heart disease, myocardial ischemia/reperfusion injury (IRI), and heart failure (HF). Severe coronary artery stenosis can lead to IRI after recanalization, HF after myocardial injury, and even ischemic cardiomyopathy (Ezekowitz et al., 2009). Therefore, a clear understanding of the pathophysiological basis behind the onset of cardiovascular diseases is of great significance for the prevention, early diagnosis, and precise treatment of cardiovascular diseases. At present, a variety of regulatory cell deaths are thought to 


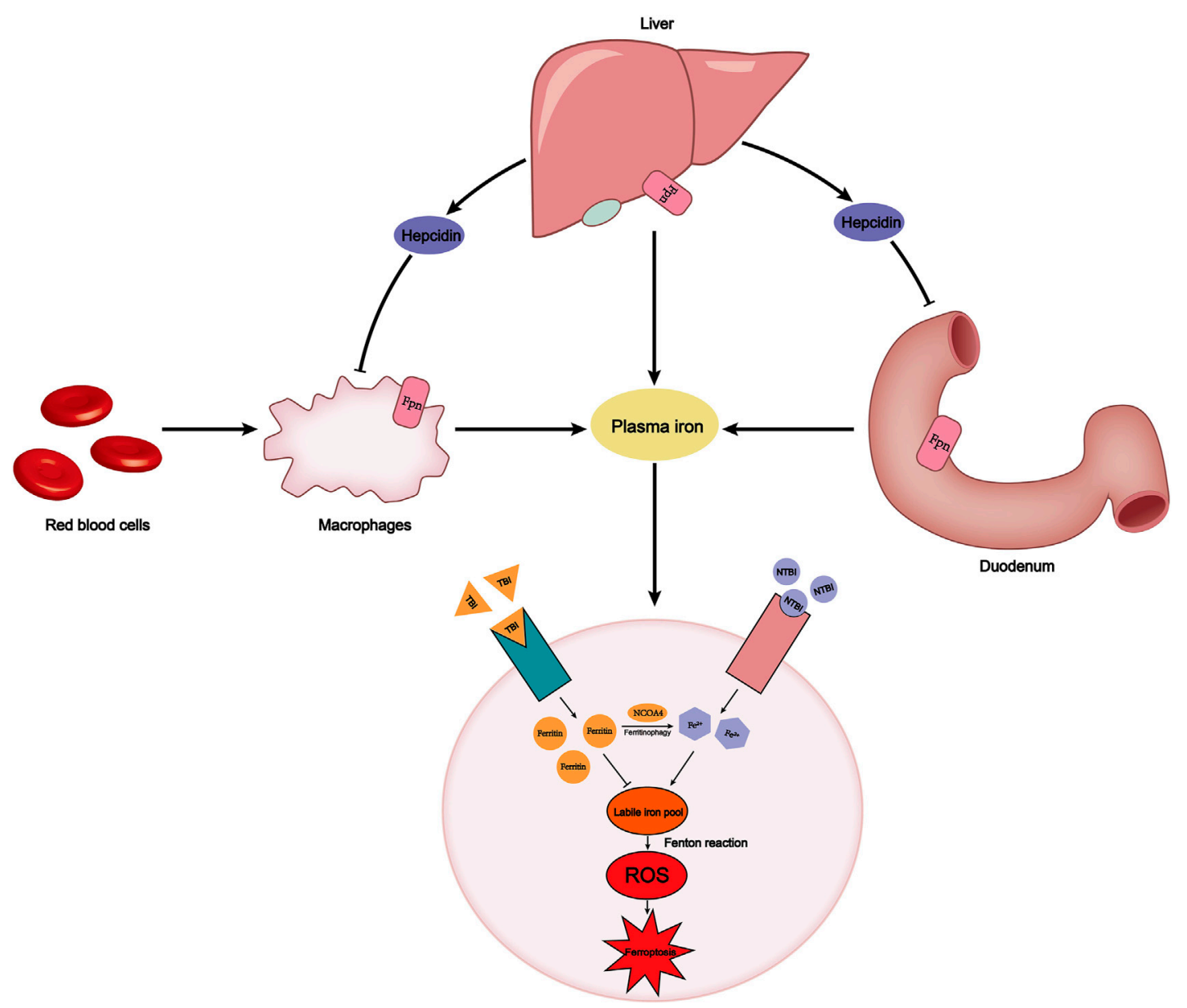

FIGURE 1 | Plasma iron concentration is always maintained within a certain range. This relies on the precise dynamic balance of iron metabolism. FPN on the surface of macrophages, the duodenum, and liver transports iron into the plasma. The endogenous source of iron is mainly hemoglobin iron that is swallowed by macrophages, while the exogenous source is iron in food absorbed by the duodenum. Hepcidin secreted by the liver can degrade FPN to reduce iron export. Plasma iron is divided into TBI and NTBI according to whether it binds to transferrin. TBI binds to the TfR1 on the cell membrane surface and stores iron in the form of ferritin, avoiding the occurrence of Fenton reaction. However, labile iron pool, derived from NCOA4-mediated ferritinophagy or NTBI absorbed by SLC39A14, will cause an increase in intracellular ROS and ferroptosis. FPN, ferroportin; TBI, transferrin-bound iron; NTBI, non-transferrin-bound iron; TfR1, transferrin receptor.

regulate the death of related cells in cardiovascular disease (Choi et al., 2019; Dong et al., 2019; Zhaolin et al., 2019). Ferroptosis, as an emerging mode of regulatory cell death, has become a new focus of research in the field of cardiovascular disease. Understanding the mechanism by which ferroptosis occurs or is inhibited in cardiovascular disease is a prerequisite to reducing morbidity and mortality in the future. In this review, we enumerate the latest developments in different aspects of the mechanism of ferroptosis and then will specifically review the unique pathological characteristics of ferroptosis in different cardiovascular diseases, which will be a reference for the prevention and treatment strategies.

\section{REGULATION OF IRON HOMEOSTASIS}

Iron is an essential trace element for the human body; it participates in the formation of a variety of proteins, including hemoglobin, myoglobin, and ferritin, and various redox reactions in cells. It is not only involved in the above-mentioned biological processes but is also the most important initiation and promotion factor for ferroptosis. Under physiological conditions, the complex and precise mechanism of iron homeostasis regulation ensures that iron concentration in the cell remains stable and prevents intracellular iron overload from triggering the Fenton reaction, which in turn leads to ferroptosis (Ganz, 2013). The regulation of iron homeostasis mainly includes mechanisms involving import, export, storage, and utilization of iron.

\section{Iron Export}

Ferroportin (Fpn), also known as SLC40A1, is the only protein known to export nonheme iron to the outside of the cell. Fpn is widely distributed on the surface of duodenal epithelial cells, macrophages, and hepatocytes. The main source of iron in the human body is hemoglobin iron recovered by macrophages phagocytosing senescent red blood cells. The macrophages 
TABLE 1 | The regulatory mechanism of ferroptosis.

\begin{tabular}{|c|c|c|c|}
\hline Target & Regulation mechanism & Effect & References \\
\hline \multirow[t]{2}{*}{ Iron export } & Hepcidin-Fpn pathway & $\begin{array}{l}\text { Hepcidin promotes FPN internalization and degradation, causing iron } \\
\text { accumulation in cells, which promotes ferroptosis }\end{array}$ & Bao et al. (2021) \\
\hline & MiR-124/Fpn pathway & $\begin{array}{l}\text { High serum levels of miR-124 inhibit FPN expression and promote } \\
\text { ferroptosis }\end{array}$ & Bao et al. (2020) \\
\hline \multirow[t]{3}{*}{ Iron import } & $\begin{array}{l}\text { Serum transferrin level, NTBI } \\
\text { and SLC39A14 }\end{array}$ & $\begin{array}{l}\text { When serum transferrin levels decrease, NTBI levels increase. NTBI } \\
\text { accelerates cellular iron accumulation and ferroptosis. But at this time, } \\
\text { SLC39A14, which is not the main transport protein under normal } \\
\text { circumstances, can antagonize the effect of NTBI. }\end{array}$ & Yu et al. (2020) \\
\hline & SREBF2 and transferrin & SREBF2 induces ferritin at the transcriptional level and inhibits ferroptosis & Hong et al. (2021) \\
\hline & USP7/P53/TfR1 pathway & $\begin{array}{l}\text { USP7 promotes P53 ubiquitination and increases TfR1 transcription. And } \\
\text { TfR1 is one of the markers of ferroptosis }\end{array}$ & Tang et al. (2021b) \\
\hline $\begin{array}{l}\text { Iron storage and } \\
\text { utilization }\end{array}$ & Ferritinophagy & $\begin{array}{l}\text { NCOA4 facilitates degradation of ferritin in a selective cargo-mediated } \\
\text { autophagy manner, which is an important source of iron for labile iron pool }\end{array}$ & Ito et al. (2021) \\
\hline $\begin{array}{l}\text { Other iron } \\
\text { metabolism }\end{array}$ & HSF1/HSPB1/TfR1 pathway & $\begin{array}{l}\text { HSF1 indirectly regulates cellular iron homeostasis and reduces the } \\
\text { expression of TfR1 through HSPB1 }\end{array}$ & Sun et al. (2015) \\
\hline \multirow[t]{2}{*}{ GPX4 } & Selenium metabolism & $\begin{array}{l}\text { Selenium not only participates in the synthesis of GPX } 4 \text { but also promotes } \\
\text { the transcription of GPX } 4 \text { through TFAP } 2 \mathrm{C} \text { and SP1 }\end{array}$ & Alim et al. (2019) \\
\hline & $\begin{array}{l}\text { mTORC1/4EBP/GPX4 } \\
\text { pathway }\end{array}$ & $\begin{array}{l}\text { MTORC } 1 \text { and 4EBP act as a bridge between cystine, cysteine and GPX4, } \\
\text { and can accelerate the synthesis of GPX } 4\end{array}$ & Zhang et al. (2021) \\
\hline \multirow[t]{5}{*}{ System $\mathrm{Xc}^{-}$} & $\begin{array}{l}\text { ATF3 and ATF4 transcriptional } \\
\text { regulation }\end{array}$ & $\begin{array}{l}\text { ATF3 binds to the SLC7A11 promoter to inhibit transcription, while ATF4 } \\
\text { does the opposite }\end{array}$ & Wang L et al. (2020), Chen et al. (2017) \\
\hline & KEAP1/NRF2/SLC7A11 & $\begin{array}{l}\text { After separation of NRF2 from KEAP1, NRF2 enters the nucleus and } \\
\text { binds with the ARE of the SLC7A11 promoter to up-regulate the } \\
\text { expression of SLC7A11 }\end{array}$ & Habib et al. (2015) \\
\hline & $\begin{array}{l}\text { BECN1 post-translational } \\
\text { modification }\end{array}$ & BECN1 directly binds to SLC7A11 to inhibit its activity & Song et al. (2018) \\
\hline & Amino acid metabolism & $\begin{array}{l}\text { AMPK/SREBP1/BCAT2 pathway reduces intracellular glutamate } \\
\text { concentration and inhibits system } \mathrm{Xc}^{-} \text {. Increasing the concentration of } \\
\text { extracellular glutamate produce the same result }\end{array}$ & Gao et al. (2015); Wang et al. (2021) \\
\hline & $\begin{array}{l}\text { Immunotherapy and } \\
\text { chemotherapy }\end{array}$ & $\begin{array}{l}\text { IFN } \gamma \text { secreted by CD8 }{ }^{+} \text {cells inhibits SLC7A11 and SLC3A2. } \\
\text { Radiotherapy can also interfere with the function of SLC7A11 }\end{array}$ & Lang et al., (2019), Wang W et al. (2019) \\
\hline $\begin{array}{l}\text { FSP1-CoQ10- } \\
\text { NADPH }\end{array}$ & MDM2 and MDMX & $\begin{array}{l}\text { The MDM2-MDMX heterodimer can up-regulate the antioxidant system } \\
\text { activity of FSP1-CoQ10 }\end{array}$ & Venkatesh et al. (2020) \\
\hline \multirow[t]{3}{*}{ Lipid metabolism } & ACSLS & $\begin{array}{l}\text { ACSL4 promotes phosphatidylethanolamine containing AA and AdA to } \\
\text { participate in phospholipid synthesis and increase the proportion of } \\
\text { phospholipids that are prone to be oxidized. And ACSL1 adjusts the lipid } \\
\text { composition by assembling aESA into DAG and TAG. }\end{array}$ & $\begin{array}{l}\text { Doll et al. (2017) } \\
\text {, Beatty et al. (2021) }\end{array}$ \\
\hline & Lipoxygenase & $\begin{array}{l}\text { ALOX12 catalyzes the oxidation of arachidonic acid. 15LOX and PEBP1 } \\
\text { combine to form a complex and then oxidize PUFA to form 15-HpETE- } \\
\text { PE, which is an important signaling molecule of ferroptosis }\end{array}$ & $\begin{array}{l}\text { Chu et al., (2019), Anthonymuthu et al. } \\
\text { (2021), Wenzel et al. (2017) }\end{array}$ \\
\hline & MDM2 and MDMX & $\begin{array}{l}\text { MDM2 and MDMX form a complex to inhibit the transcription factor } \\
\text { PPARa, and up-regulate the sensitivity to ferroptosis }\end{array}$ & Venkatesh et al. (2020) \\
\hline
\end{tabular}

FPN, ferroportin; NTBI, non-transferrin-bound iron; USP7, ubiquitin-specific protease 7; TfR1, transferrin receptor 1; HSF1, heat shock factor 1; HSPB1, heat shock protein beta-1; GPX4, glutathione peroxidase 4; mTORC1, mechanistic target of rapamycin complex 1; ATF3, activating transcription factor 3; ATF4, activating transcription factor 4; KEAP1, Kelch-like echassociated protein 1; NRF2, NF E2 Related Factor 2; ARE, antioxidant response element; AMPK, AMP-activated protein kinase; SREBP1, sterol response element binding protein 1; BCAT2, branched-chain amino acid aminotransferase 2; IFN $\gamma$, interferon $\gamma$; FSP1, Ferrop-Suppressor-Protein 1; ACSLs, acyl-CoA synthetase long-chain family; ACSL4, long-chain acylCOA synthetase 4; AA, arachidonic acid; AdA, adrenal acid; ACSL1, long-chain acyl-CoA synthetase 1; DAG, diacylglycerols; TAG, triacylglycerols; ALOX12, arachidonate 12lipoxygenase; 15LOX, 15-lipoxygenase; PEBP1, phospholipid-ethanolamine binding protein-1; PUFA, polyunsaturated fatty acid; 15-HpETE-PE, 15-hydroperoxy-eicosatetraenoyl phosphatidylethanolamine.

then transport the iron into plasma through Fpn. Simultaneously, the duodenum absorbs iron ions from food into the human body through Fpn (Figure 1) (Ganz, 2013). The lack of Fpn on the membrane surface of other tissues leads to intracellular iron enrichment (Donovan et al., 2005). Bao et al. found that Fpn was down-regulated, leading to accumulation of lipid peroxide and neuronal cell ferroptosis in a mouse model of Alzheimer's disease (Table 1) (Bao et al., 2021). Hepcidin, a hepatic-secreted peptide hormone, binds to Fpn and promotes internalization and degradation of Fpn, resulting in the decreased export of nonheme iron (Nemeth et al., 2004). In addition to the Hepcidin-Fpn signaling pathway, the miR-124/Fpn signaling pathway has also been confirmed to affect the occurrence of ferroptosis. High levels of miR-124 in serum inhibit the expression of Fpn, leading to iron accumulation and ferroptosis in cells (Bao et al., 2020).

\section{Iron Import}

Under physiological conditions, ferric iron exported from cells binds to transferrin, which is a protein secreted primarily by the liver and transports iron to the bone marrow and other tissues. Conversely, if transferrin in plasma decreases, iron binds to albumin and anions to form non-transferrin-bound iron 
(NTBI), which can be absorbed by tissue cells, causing iron overload (Ganz, 2013). Yu et al. reported that when transferrin levels in serum decreased, the vulnerability of liver fibrosis in both humans and mice increased significantly, which was due to the accumulation of NTBI in liver cells and leads to ferroptosis of liver cells. In this case, SLC39A14, which is not the main transporter under physiological conditions, can act as a transporter for NTBI to induce ferroptosis (Yu et al., 2020). However, research has found that SREBF2, the principal regulator of cholesterol synthesis, can directly induce transferrin expression at the transcription level, reduce intracellular iron pools, and suppress ferroptosis induced by iron accumulation (Hong et al., 2021). Transferrin receptor 1 (TfR1) can transfer transferrin into cells through receptormediated endocytosis, and release iron carried by transferrin into the iron pool. TfR 1 has been identified as a specific target antigen related to ferroptosis (Feng et al., 2020). The research by Tang et al. (2021a) demonstrated that Ubiquitin-specific protease 7 (USP7) promotes ubiquitination of P53, leading to increased transcription of TfR1 and ferroptosis (Tang et al., 2021a).

\section{Iron Storage and Utilization}

Ferritin is an intracellular protein that oxidizes ferrous iron to ferric iron to avoid the occurrence of Fenton reaction, composed of light subunits (Ftl) and heavy subunits (Fth1). Ferritin is an inhibitor of ferroptosis, knockdown of Fth1 expression significantly increases the occurrence of ferroptosis (Rui et al., 2021). Ferritin is a form of iron storage in cells, and ferroptosis requires ferritin degradation to release stored iron. Quantitative proteomics found that NCOA4 can deliver ferritin to lysosomes and degrade ferritin in a selective cargo-mediated autophagy manner. Therefore, this process is called ferritinophagy (Mancias et al., 2014). Iron-independent ROS in the cell induces ferritinophagy and will increase intracellular iron levels (Park and Chung, 2019). The ferrous ions produced by ferritinophagy and the NTBI participate in the formation of labile iron pool in the cell, which is the chief culprit for the generation of ROS through the Fenton reaction (Ito et al., 2021). Therefore, iron storage and iron utilization are involved in the occurrence of ferroptosis.

\section{Other Iron Metabolism}

Novel regulators of iron homeostasis have been described. Sun et al. found that phosphorylated heat shock protein beta1(HSPB1) acted as a negative regulator of ferroptosis by reducing iron uptake by cells and the production of lipids. Transphosphorylation of HSPB1 increases ferritin expression and reduces TfR1 expression. HSPB1 is expressed in a heat shock factor 1(HSF1) dependent manner. Protein kinase C (PKC) regulates the phosphorylation of HSPB1 and indirectly regulates ferroptosis (Sun et al., 2015). The HSF1-HSPB1 pathway and $\mathrm{PKC}$ are novel regulators of iron homeostasis.

\section{ANTIOXIDANT METABOLISM}

\section{Glutathione Peroxidase 4}

Not only does abnormal iron homeostasis regulation play an important role in ferroptosis but ferroptosis also requires the dysregulation of the intracellular antioxidant system. Yang et al. found that in ferroptosis cells, reduced glutathione (GSH), one of the most important endogenous antioxidants, was depleted, leading to the inactivation of glutathione peroxidase 4 (GPX4) (Yang et al., 2014). GSH-dependent GPX4 transforms toxic lipid peroxides in cells into non-toxic fatty alcohols, and prevents membrane phospholipids from reacting with ROS to produce lipid peroxides that directly lead to ferroptosis (Figure 2) (Magtanong et al., 2016). GSH is an essential substrate for GPX4 and exerts antiferroptosis activity. It is synthesized from cysteine and glutamic acid through a two-step enzymatic reaction and is oxidized to oxidized glutathione (GSSG) after reacting with lipid peroxides. Therefore, the antioxidant system comprising the GPX4 core is considered one of the most powerful regulators for protecting cells from ferroptosis. The appearance of a variety of diseases has been closely associated with the knockout of GPX4, such as neuronal degeneration (Hambright et al., 2017) and acute renal failure (Friedmann Angeli et al., 2014). Conversely, inhibiting GPX4 in drug-tolerant persisted cancer cells increases their vulnerability to ferroptosis. This discovery suggests a new treatment strategy for tumors (Hangauer et al., 2017; Viswanathan et al., 2017).

Selenoproteins are unique and rare proteins among a large protein family with unique physiological functions. Selenocysteine, which is not one of the 20 proteinogenic amino acids, is involved in the synthesis of GPX4. Selenocysteine ensures the need for unexpected antioxidant capacity (Ingold et al., 2018). Moreover, selenium is not only related to the synthesis of GPX4 but also promotes GPX4 transcription by coordinating and activating the transcription factors TFAP2C and SP1, allowing cells to respond to ferroptosis stimulation in an adaptive manner (Alim et al., 2019). In addition to the regulation of the transcription level, cystine and cysteine, as raw materials that simultaneously participate in the synthesis of GSH and GPX4, can activate the mechanistic target of rapamycin complex 1 (mTORC1) to inhibit the downstream 4EBP axis and promote GPX4 synthesis instead of indirectly regulating GSH levels (Zhang et al., 2021). In fact, mTORC1 acts as a bridge to link cystine and cysteine availability with the regulation of GPX4 and ferroptosis. Gaschler et al. recently discovered a new type of ferroptosis inducer FINO2 that inhibits GPX4. This is different from the class II ferroptosis inducer represented by RSL3. FINO2 can inhibit GPX4 while directly oxidizing polyunsaturated fatty acids (PUFAs), and provides a new perspective for the intervention of ferroptosis by adjusting GPX4 levels (Gaschler et al., 2018).

\section{System Xc-}

System $\mathrm{Xc}^{-}$is an amino acid reverse transporter located on the surface of the cell membrane, which can transport cystine outside the cell and glutamate inside the cell in a 1:1 ratio across the membrane. Therefore, System $\mathrm{Xc}^{-}$activity is closely related to intracellular GSH content and GPX4 function. Ferroptosis is induced if system $\mathrm{Xc}^{-}$activity is inhibited by erastin treatment (Dixon et al., 2012; Dixon et al., 2014). System $\mathrm{Xc}^{-}$is a dimer composed of two subunits, SLC7A11 and SLC3A2, of which SLC7A11 makes a critical difference in limiting the rate of amino 


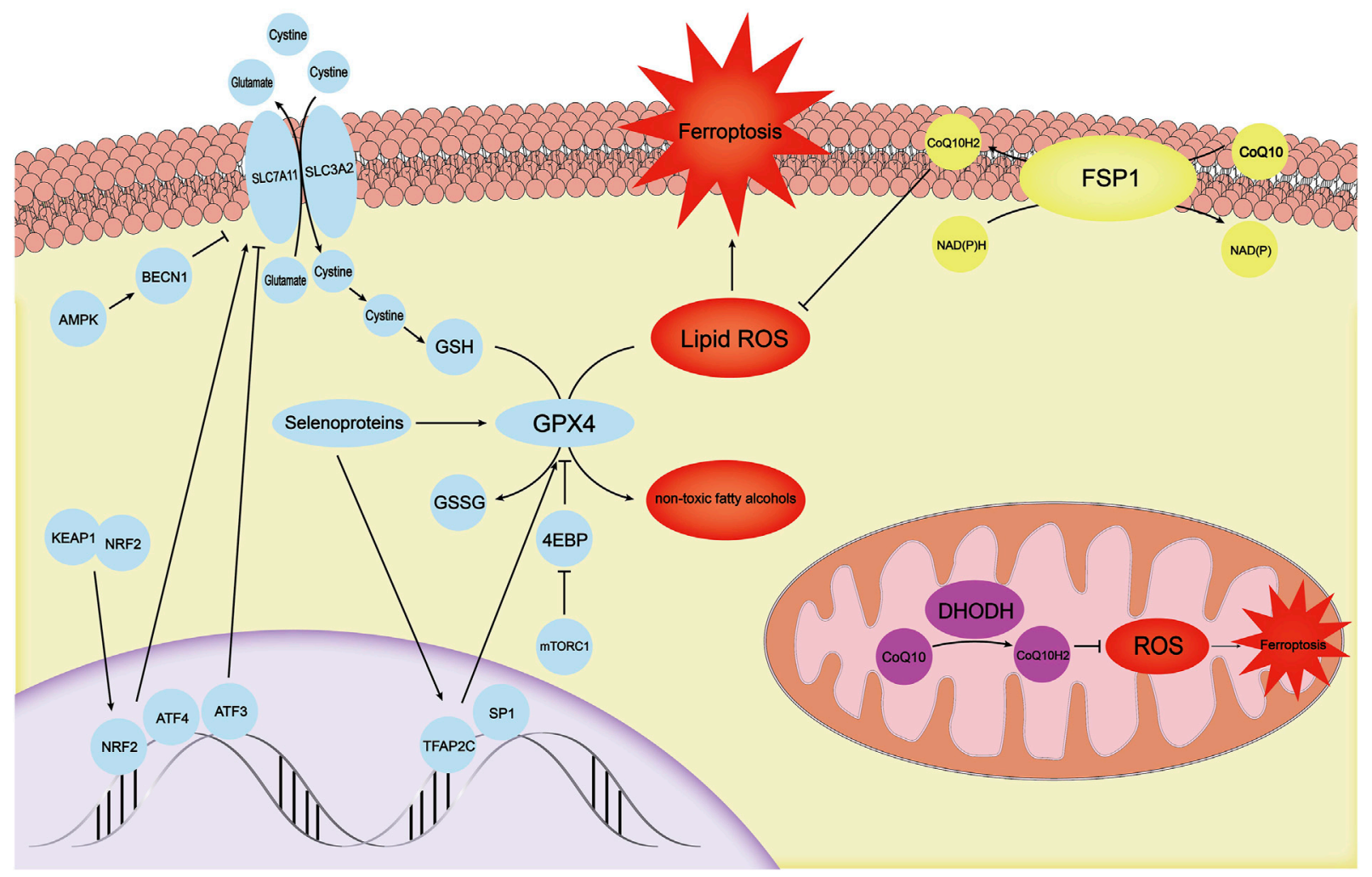

FIGURE 2 | There are three independent antioxidant systems in cells. The antioxidant system with GPX4 as the core is the first antioxidant system discovered. It is regulated by mechanisms such as TFAP2C, SP1, mTOR-4EBP, and selenoproteins. SLC7A11, which provides a substrate for GPX4, is regulated by ATF3, ATF4, KEAP1-NRF2, and AMPK-BECN1. The FSP1-CoQ10-NADPH system, another antioxidant system in the cell membrane, can inhibit ferroptosis through CoQ10H2. DHODH primarily inhibits ROS in mitochondria by synthesizing $\mathrm{CoQ} 10 \mathrm{H} 2$ to avoid the ferroptosis process in mitochondria. GPX4, glutathione peroxidase 4; mTOR, mechanistic target of the rapamycin complex 1; ATF3, activating transcription factor 3; ATF4, activating transcription factor 4; KEAP1, kelch-like ech-associated protein 1; NRF2, NF E2 Related Factor 2; AMPK, AMP-activated protein kinase; FSP1, Ferrop-Suppressor-Protein 1; NADPH, nicotinamide adenine dinucleotide phosphate; $\mathrm{DHODH}$, dihydroorotate dehydrogenase; ROS, reactive oxygen species.

acid transport (Lewerenz et al., 2013). Multiple regulatory mechanisms at transcription, post-translational modification (PTMS), and protein levels determine the ability of System $\mathrm{Xc}^{-}$to uptake cystine.

Activating transcription factors are a family of transcription factors that includes activating transcription factor 3 (ATF3) and activating transcription factor 4 (ATF4), which have been confirmed to be related to the function of the system $\mathrm{Xc}^{-}$ (Wang J et al., 2020; Chen et al., 2017). In the presence of the intracellular redox imbalance, the mRNA level of ATF3 increased. Activated ATF3 binds tightly to the SLC7A11 promoter, inhibits the expression of SLC7A11, and promotes the occurrence of ferroptosis in oxidatively stressed cells (Wang L et al., 2020). Interestingly, the effects of ATF4 is completely opposite. Increased expression of ATF4 up-regulates system $\mathrm{Xc}^{-}$expression and increases cell resistance to ferroptosis (Chen et al., 2017). There are other transcription factors, such as the redox-sensitive transcription factor NF E2 Related Factor 2 (NRF2) and P53, which regulate the function of system $\mathrm{Xc}^{-}$ (Habib et al., 2015). In the basal state, Kelch-like ech-associated protein 1 (KEAP1) ubiquitinates NRF2, and when cells are under oxidative stress, NRF2 separates from KEAP1. Subsequently,
NRF2 enters the nucleus and binds to the antioxidant response element (ARE) of the proximal promoter of its target gene SLC7A11 to up-regulate the expression of SLC7A11 (Habib et al., 2015). As the most famous tumor suppressor gene, P53 has unique and diverse functions in the regulation of ferroptosis. Under stimulation of low or moderate levels of ROS, P53 can prevent the accumulation of lethal ROS, but when high levels of ROS are produced, P53 causes ferroptosis in cells by inhibiting SLC7A11 transcription (Jiang et al., 2015). Multiple transcription factors can be considered indirect regulatory targets of ferroptosis.

Unlike regulation at the transcriptional level, BECN1 can regulate the activity of system $\mathrm{Xc}^{-}$through post-translational modification. BECN1 binds directly to SLC7A11 to form the BECN1-SLC7A11 complex, rather than interfering with the transcription of SLC7A11. Prior to this, BECN1 requires phosphorylation at Ser90/93/96 via AMP-activated protein kinase (AMPK) (Song et al., 2018).

Amino acid metabolism is the main mechanism regulating protein levels, and both cysteine and glutamate within and outside the cell have an impact on system $\mathrm{Xc}^{-}$. High extracellular glutamate concentrations inhibit the activity of 
system $\mathrm{Xc}^{-}$, whereas elevated intracellular glutamate concentrations have the opposite effect. If cells are treated with erastin or sorafenib, the intracellular labile iron level will increase and subsequently induce ferritinophagy (Park and Chung, 2019). Wang et al. showed that ferritinophagy activated downstream AMPK and inhibited nuclear translocation of the sterol response element binding protein 1 (SREBP1), resulting in reduced transcription of the branchedchain amino acid aminotransferase 2 (BCAT2). BCAT2 is involved in the de novo synthesis of intracellular glutamate, and inhibition of BCAT2 reduces intracellular glutamate, which induces ferroptosis in cells (Wang et al., 2021). Similarly, inhibition of extracellular glutamine breakdown, which creates extracellular conditions of high glutamate, inhibits ferroptosis (Gao et al., 2015).

Recently, unique mechanisms by which immunotherapy and chemotherapy affect the system $\mathrm{Xc}^{-}$have been reported. It was found that immune-activated $\mathrm{CD}{ }^{+} \mathrm{T}$ cells secreted interferon $\gamma$ $(\mathrm{IFN} \gamma)$ that downregulated the expression of SLC7A11 and SLC3A2 expression (Lang et al., 2019; Wang W et al., 2019). SLC7A11 has also been shown to be a key target for the simultaneous action of immunotherapy and radiation therapy to induce ferroptosis (Lang et al., 2019). These novel studies provide a new research perspective to explore the mechanism of regulation of the system $\mathrm{Xc}^{-}$, and future studies may focus on the effect of the intervention of physiological factors.

\section{Other Parallel Antioxidant Mechanisms}

Do cells have only one barrier against ferroptosis? Whether there is a parallel but completely independent antioxidant system with GPX4 and system $\mathrm{Xc}^{-}$has become the key question regarding its antioxidant mechanism. Two research groups, Doll et al. (2019) and Bersuker et al. (2019) described independent mechanisms for cell resistance to ferroptosis almost simultaneously. The researchers determined that in some tumor cell lines, cells were able to resist ferroptosis even when the antiferroptosis system with the core of GPX4 was absent. Through gene sequencing of a large number of cells, the authors identified a previously unrecognized new mitochondrial-associated 2 apoptosis inducing factor (AIFM2), and renamed it "FerropSuppressor-Protein 1" (FSP1). FSP1 must be recruited to the lipid membrane after myristoylation to perform its reductase function (Bersuker et al., 2019). The lipid membrane is rich in ubiquinone (CoQ10), and its reduced form, ubiquinol, can act as a lipophilic radical that traps antioxidant harvesting lipid peroxyl radicals (Doll et al., 2019). To reduce CoQ10, FSP1 must be supported by the coenzyme nicotinamide dinucleotide phosphate (NADPH), so FSP1-CoQ10-NADPH constitutes an independent antiferroptotic system.

In fact, cells contain other parallel antioxidant mechanisms. According to a recent study by Mao et al., there is a unique ferroptosis resistance system in mitochondria, which is dominated by dihydroorotate dehydrogenase (DHODH) (Mao et al., 2021). DHODH in GPX4-deficient cells leads to significant mitochondrial lipid peroxidation. Similarly, to the activity of the FSP1-CoQ10-NADPH axis, DHODH promotes the regeneration of CoQ10, which traps lipid peroxides in the mitochondrial membrane. Intervention with other parallel antioxidant mechanisms is a promising target to inhibit ferroptosis.

\section{LIPID METABOLISM}

The Fenton reaction triggered by abnormal iron metabolism leads to an increase in ROS, and an antioxidant system represented by GPX4 can eliminate ROS toxicity to cells, but the link between the two is lipid metabolism within the cell. Phospholipids are a component of cell membranes. ROS generated by the Fenton reaction tends to react with phospholipids (PL) to form hydroperoxy-phospholipids (PLOOH), which are the main elimination target of GPX4 (Magtanong et al., 2016). Phospholipids are lipids containing phosphoric acid, including a hydrophobic part composed of fatty acids and a hydrophilic part containing phosphoric acid. Phospholipids composed of polyunsaturated fatty acids are the main substrate for ROS oxidation (Kagan et al., 2017). Polyunsaturated fatty acids refer to straight chain fatty acids containing two or more double bonds, divided mainly into the omega- 6 and omega- 3 series (Wiktorowska-Owczarek et al., 2015). In the process of lipid synthesis, the first reaction using fatty acids is to catalyze the conversion of fatty acids into fatty acyl-CoA. The acyl-CoA synthetase long-chain family (ACSLs) is the first enzyme to catalyze the synthesis of phospholipids by acyl-CoA. Among them, long-chain acyl-CoA synthetase 4 (ACSL4) was identified as the key executor of ferroptosis (Yuan et al., 2016).

The preferred substrate for oxidation is phosphatidylethanolamine containing arachidonic acid (AA) and adrenal acid (AdA), and ACSL4 has a strong selectivity for AA-CoA and AdA-CoA (Doll et al., 2017; Dierge et al., 2021). ACSL4 promotes more peroxidable fatty acids to participate in the phospholipid synthesis process, and changes the composition ratio of fatty acids in lipids (Doll et al., 2017). Long-chain acylCoA synthetase 1 (ACSL1) adjusts the lipid composition by assembling aESA into diacylglycerols (DAG) and triacylglycerols (TAG). ACSL4 and ACSL1 adjust the content of non-conjugated fatty acids and conjugated fatty acids in lipids to adjust the vulnerability to ferroptosis. Unlike ACSL, lipoxygenase consists of a series of enzymes that catalyze the oxidation of PUFA double bonds to form lipid peroxides. Arachidonate 12-lipoxygenase (ALOX12) mainly catalyzes the oxidation of arachidonic acid, which is closely related to P53mediated ferroptosis (Chu et al., 2019). P53 can indirectly activate ALOX12 by inhibiting SLC7A11 transcription, causing ferroptosis. It should be noted that although the transcription of SLC7A11 is reduced, it does not have a significant effect on GPX4 and GSH, indicating that this is an independent ferroptosis mechanism of GPX4 and ACSL4 (Chu et al., 2019), which involves 15-lipoxygenase (15LOX) and phospholipidethanolamine binding protein-1 (PEBP1) to combine and form a complex. The 15LOX/PEBP1 complex oxidizes various PUFAs to form 15-hydroperoxy-eicosatetraenoyl phosphatidylethanolamine (15-HpETE-PE), an important signaling molecule of ferroptosis (Wenzel et al., 2017; Anthonymuthu et al., 2021). 


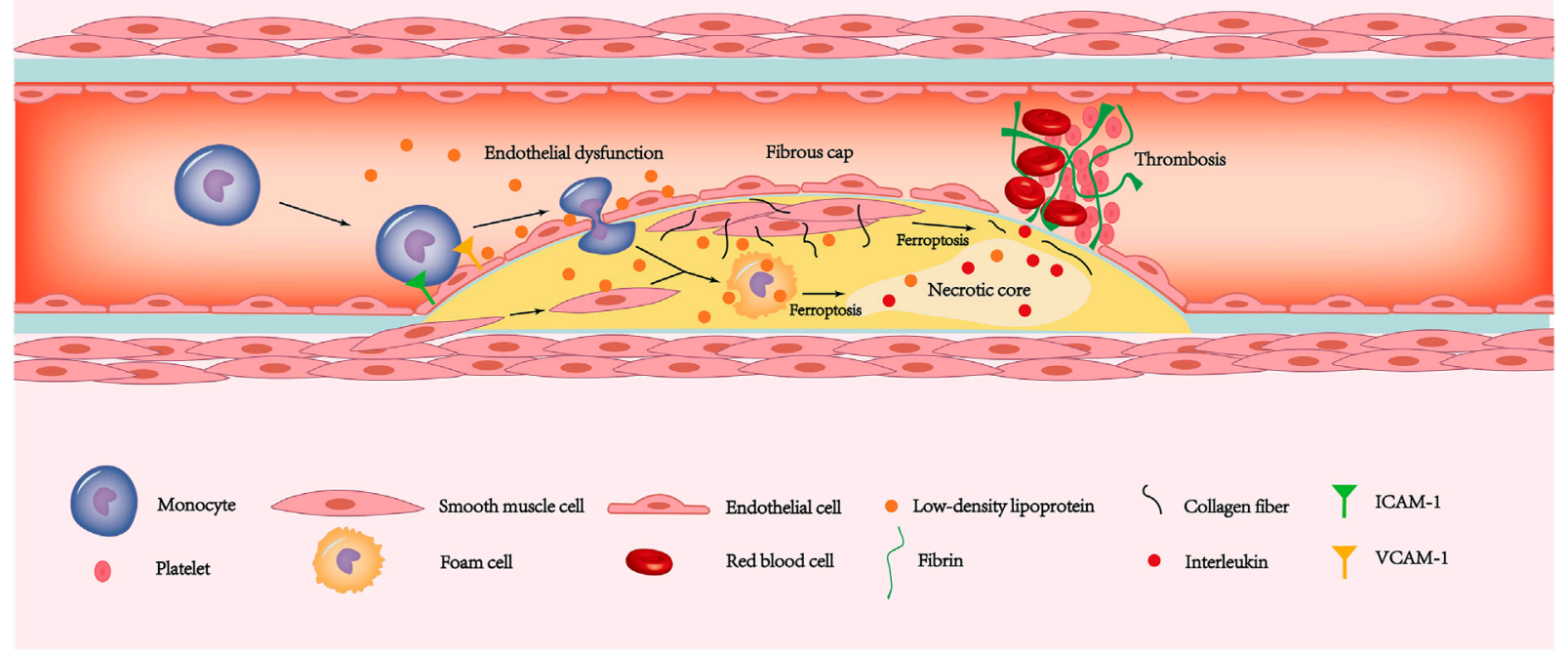

FIGURE 3 | The ferroptosis of endothelial cells, smooth muscle cells and macrophages is involved in the pathogenesis of coronary atherosclerotic heart disease. The increased expression of ICAM-1 and VCAM-1 allows monocytes to adhere to the endothelial surface. Due to ferroptosis leading to endothelial dysfunction, monocytes have the opportunity to deform and enter the inner membrane. The monocytes under the inner membrane gradually transform into macrophages. Then some macrophages and smooth muscle cells phagocytose LDL and then form foam cells. In addition, some smooth muscle cells migrate to the plaque surface and secrete a large amount of extracellular matrix such as collagen fibers, forming thick fibrous caps. As the disease progresses, ferroptosis in smooth muscle cells leads to gradual thinning of the fibrous cap. Similarly, foam cells ferroptosis promotes the release of interleukins in the necrotic core. Exfoliation of the endothelium caused by ferroptosis exposes fibrinogen to the tissue. The combination of thin fibrous caps, endothelial stripping, and large amounts of interleukin released from the necrotic core leads to a rapidly forming thrombosis that blocks the coronary artery and causes ACS. LDL, low-density lipoprotein; ACS, acute coronary syndrome.

MDM2 and MDMX are negative regulatory proteins of the P53 gene. However, their role in ferroptosis has nothing to do with P53, but with lipid metabolism (Venkatesh et al., 2020). During ferroptosis, the MDM2-MDMX heterodimer formed by MDM2 and MDMX inhibits the activity of the transcription factor PPARa in a post-translational manner. The PPAR transcription factor family is a large-scale regulator of lipid components, and reduced PPARa activity increases susceptibility to ferroptosis. At the same time, the MDM2MDMX heterodimer can up-regulate the antioxidant system activity of FSP1-CoQ10 (Venkatesh et al., 2020). In conclusion, the lipid composition of the cell membrane is both the substrate and site of ferroptosis execution, and the regulation of the lipid composition of the cell membrane is an independent mechanism able to regulate ferroptosis.

\section{FERROPTOSIS AND CORONARY ATHEROSCLEROTIC HEART DISEASE}

Coronary artery disease refers to a series of diseases with angina pectoris as the main symptom caused by the inability of the coronary blood supply to meet the metabolic needs of the myocardium. Coronary artery disease is a general term for a series of diseases that include coronary atherosclerotic heart disease, coronary artery spasm, and myocardial bridge-of which coronary atherosclerotic heart disease accounts for the vast majority. Coronary atherosclerotic heart disease is a disease characterized by hyperplasia of atherosclerotic plaques. In the early stage of atherosclerosis, endothelial cell ability to resist blood cell attachment is reduced under stimulation of high levels of low-density lipoprotein (LDL) (Verhoye and Langlois, 2009; Trpkovic et al., 2015), hypertension (Quyyumi and Patel, 2010) and inflammation (Weissberg and Bennett, 1999). The expression of adhesion molecules on the surface of endothelial cells is dramatically increased, and the permeability of endothelial cells and the subendothelial extracellular matrix are simultaneously altered (Figure 3). This process is called endothelial dysfunction. Circulating monocytes adhere to the surface of the artery intima under the impact of endothelial cell adhesion molecules and gradually penetrate the arterial intima (Libby et al., 2011). Monocytes entering the intima differentiate into mononuclear macrophages under stimulation of inflammatory factors. Under stimulation of inflammation, the smooth muscle cells of the arterial media are recruited into the intima and merge with smooth muscle cells that settle in the inner membrane (Libby et al., 2011).

Endothelial dysfunction also causes the lipid composition of the blood to gradually accumulate under the inner membrane. To remove deposited lipids, especially LDL, monocytes, macrophages, and smooth muscle cells will phagocytose lipids to form foam cells. As lipids and foam cells accumulate and lipid deposition under the inner membrane, a fatty streak gradually forms in the intima, which are the early lesions of atherosclerosis (Maguire et al., 2019; Wang D et al., 2019). However, the phagocytic ability of foam cells is limited and the deposited lipid cannot be engulfed completely. Therefore, a large number of cell components are wrapped by the lipid pool 
formed by extracellular lipids, and these components together become the basis for the formation of the lipid core. Long-term hypoxia and inflammation in the lipid core cause foam cells to die in a variety of ways, including necrosis and apoptosis. This process releases components such as cell debris, which constitute the main component of the necrotic core (Tabas, 2010). Smooth muscle cells secrete a large amount of extracellular matrix, including collagen fibers and elastin, covering the surface of the necrotic core, which is called the fibrous cap. The necrotic core and fibrous cap together constitute the pathological basis of coronary atherosclerotic heart disease and coronary atherosclerotic plaque formation.

As plaque size increases, coronary lumen stenosis reduces blood supply to cardiomyocytes and presents as angina pectoris. A potentially more lethal process may occur if the plaque thrombus suddenly occludes the coronary arteries and blood flow is drastically reduced. The produces a lack of collateral circulation compensation and can cause acute coronary syndromes (ACS), including unstable angina (UA) and acute myocardial infarction (AMI) (Crea and Libby, 2017). According to current opinions, the causes of acute thrombosis on the plaque surface are divided mainly into two types: plaque rupture (Davies and Thomas, 1984) and plaque erosion (Dai et al., 2018). Plaque rupture is the main cause of ACS. About two-thirds of ACSs are caused by plaque rupture (Arbustini et al., 1999). Plaques that are prone to rupture are called vulnerable plaques. Pathological features of vulnerable plaques include thin fibrous caps, large necrotic cores, and few smooth muscle cells (Finn et al., 2010). Macrophages cause vulnerable plaque rupture and thrombosis through a variety of regulatory cell death methods, including apoptosis (Hutter et al., 2004) and necroptosis (Karunakaran et al., 2016). Unlike macrophages, smooth muscle cells secrete an extracellular matrix to protect vulnerable plaques. The reduction in smooth muscle cells caused by multiple cell death pathways can significantly increase plaque vulnerability (Bennett et al., 2016).

Another vital pathogen that causes the occurrence of ACS is plaque erosion, which is responsible for up to about a third of ACS incidence. Compared to plaque rupture, plaque erosion has smaller necrotic cores, a higher number of smooth muscle cells, and thicker fibrous caps (Crea and Libby, 2017). In fact, the current explanation for the mechanism of plaque erosion is the two-hit hypothesis. De-endothelization of the plaque surface is the initial step of plaque erosion, which is the first hit. Endothelial cell dysfunction or death leads to the activation of platelets in the blood and the release of neutrophil extracellular traps (NETs) by neutrophils (Döring et al., 2017). The above factors are called the second hit, which induces thrombosis on the surface of the plaque. Through the study of the atherosclerosis process, it is easy to see that the death of various cells plays a key role in the occurrence and development of atherosclerosis. Current studies have confirmed that RCD methods such as apoptosis (Hutter et al., 2004), necroptosis (Karunakaran et al., 2016), and pyrolysis (Zhou et al., 2021) all occur in the plaque evolution process. Thus, do macrophages, smooth muscle cells and endothelial cells undergo ferroptosis during plaque progression?

As early as 30 years ago, Sullivan proposed the iron hypothesis, which pointed out that iron accumulation in atherosclerosis increases the risk of cardiovascular disease (Sullivan, 1981). The iron hypothesis is still controversial because the mechanism of iron accumulation leading to coronary atherosclerotic heart disease is not yet fully understood. The iron hypothesis has been widely questioned because hereditary hemochromatosis, a disease characterized by iron deposition throughout the body, not only reduces the risk of coronary atherosclerotic heart disease but also reduces the incidence (Mascitelli and Goldstein, 2014). Recent studies have shown that HFE gene mutations in patients with hemochromatosis regulate the expression of LDL receptors in liver cells and Kupffer cells. Under iron stimulation, HFE-deficient Kupffer cells phagocytose LDL-C in large amounts and reduce blood lipid levels (Demetz et al., 2020). Therefore, the reduced risk of coronary atherosclerosis in patients with hemochromatosis patients is actually related to the secondary low plasma level of LDL-C, which once again perfects and verifies the correctness of the iron hypothesis. Since the iron hypothesis was proposed, it has been widely recognized after continuous improvement, but the specific mechanism of iron deposition leading to atherosclerosis has not yet been fully elucidated.

The discovery of ferroptosis may explain the biological effects of iron accumulation. A recent study found that the SIRT1 autophagy axis in foam cells can inhibit ferroptosis induced by excessive iron (Su et al., 2021). By adding the exogenous iron agent ferric ammonium citrate to THP-1 cells, Su et al. confirmed that excessive iron induced ferroptosis in foam cells. Excess iron inhibits the SIRT1-autophagy axis of foam cells and reduces GPX4, both of which together induce foam cell ferroptosis ( $\mathrm{Su}$ et al., 2021). Ferroptosis foam cells will release large amounts of inflammatory mediators such as IL- $1 \beta$ and IL-18. IL- $1 \beta$ and IL-18 belong to the IL-1 family of cytokines, and both are processed by caspase-1 for their activation (Pfeiler et al., 2019). Furthermore, early studies have confirmed that IL-1 $\beta$ and IL-18 can not only promote the progression of atherosclerosis (Mallat et al., 2001; Kirii et al., 2003) but also cause late plaque instability (Mallat et al., 2001) and induce myocardial infarction (Seta et al., 2000). Therefore, macrophage ferroptosis and foam cells derived from macrophage ferroptosis are present throughout the process, from early plaque formation to late plaque instability.

Regulating macrophage ferroptosis and foam cells derived from macrophages has become a novel target for the treatment of coronary atherosclerotic heart disease. Iron is a necessary condition for macrophage ferroptosis, and the most important pathway for macrophage iron regulation is the hepcidin-FPN axis (Wunderer et al., 2020). Hepcidin degrades FPN on the surface of macrophages, the only iron export protein, thus increasing the iron content of macrophages (Donovan et al., 2005). If hepcidin expression is suppressed, macrophages can excrete excess iron and cholesterol, which reduces foam cell formation and the progression of atherosclerosis (Saeed et al., 2012). When the iron content is increased or under inflammatory conditions, bone morphogenetic protein (BMP) stimulates the expression of hepcidin. Similarly, inhibition of BMP can also significantly delay atherosclerosis and vascular calcification (Derwall et al., 2012). Although current studies have confirmed the two facts that macrophage ferroptosis promotes 
the progression of atherosclerosis and hepcidin interferes with the process of atherosclerosis by regulating iron content, the link between macrophage ferroptosis and hepcidin has not been confirmed. Whether hepcidin can affect the development of atherosclerosis by regulating macrophage ferroptosis has become a current research hotspot.

Smooth muscle cells are the cellular components of the fatty streak and the most important source of extracellular matrix secretion in plaques. Unlike macrophages, smooth muscle cell death interferes mainly with plaque vulnerability by affecting the components of the fibrous cap on the surface of the plaque (Bennett et al., 2016). Smooth muscle cells secrete collagen fibers, the main component of the fibrous cap. As smooth muscle cells die through various pathways, the thickness of the fibrous cap becomes thinner due to reduced secretion and degradation of collagen fibers (Allahverdian et al., 2018). Thin cap fibrous atheroma is usually responsible for acute cardiovascular events. Therefore, when smooth muscle cells die in the form of ferroptosis caused by various triggers, such as smoking and diabetes, it is likely to promote plaque rupture and lead to ACS. Sampilvanjil et al. extracted the components of the gas phase of cigarettes, which are the main components of cigarettes that induce cardiovascular disease, mainly including acrolein and methyl vinyl ketone (Sampilvanjil et al., 2020). Smooth muscle cells treated with components of the gas phase of cigarettes showed obvious ferroptosis characteristics, such as increased expression of PTGS2, increased active oxygen content of lipids, and depletion of GSH. Smooth muscle cells overexpressing GPX4 cannot escape ferroptosis induced by gas phase extracts. However, iron chelators and N-Acetyl-L-cysteine (an antioxidant that can replace GSH) can prevent ferroptosis of smooth muscle cells, demonstrating that cigarettes can induce ferroptosis by depleting GSH within smooth muscle cells (Sampilvanjil et al., 2020). Moreover, ferroptosis can also promote vascular calcification to cause coronary atherosclerotic heart disease (Durham et al., 2018).

Coronary artery calcification is an independent risk factor for coronary atherosclerotic heart disease and is closely related to plaque rupture (Nicoll and Henein, 2014). Smoking, diabetes, metabolic syndrome, chronic kidney disease, and oxidative stress are closely related to smooth muscle cell-induced vascular calcification (Durham et al., 2018). Oxidative stress has been identified as an initiating factor for smooth muscle cell-mediated phenotypic transformation and calcium salt secretion (Byon et al., 2008). Ma et al. used palmitic acid to stimulate smooth muscle cells to construct an in vitro model of oxidative stress, and found that smooth muscle cells produce ferroptosis and promote calcification deposition (Ma et al., 2021). In terms of mechanism, the extracellular matrix protein periostin (POSTN) negatively regulates the expression of SLC7A11 by inhibiting the expression of the smooth muscle cell P53 gene. After pretreatment with metformin, the ferroptosis of smooth muscle cells was significantly reduced, and the calcification deposition disappeared. In conclusion, metformin activates the antioxidant system of smooth muscle cells to resist ferroptosis and secondary calcification through the Nrf2 signaling pathway, which finally confirmed the close connection between ferroptosis and vascular calcification. Preventing coronary heart disease by intervening in smooth muscle cell ferroptosis will become another promising independent therapeutic target.

It was reported that endothelial cell ferroptosis contributes to the activation of adhesion factor and thrombus formation. The mechanism that triggers endothelial dysfunction is mainly related to endothelial cell oxidative stress, and ferroptosis also plays a role in oxidized low-density lipoprotein (oxLDL)-induced endothelial cell dysfunction (Bai et al., 2020). When endothelial cell dysfunction occurs, adhesion molecules on the surface of endothelial cells are overexpressed, including the intercellular adhesion molecule-1 (ICAM-1) and the vascular cell adhesion molecule-1 (VCAM-1) (Habas and Shang, 2018). The ferroptosis inhibitor Fer-1 reversed ICAM-1 and VCAM-1 expression secondary to the reduction in ferroptosis of endothelial cells and restored vascular endothelial cell function. GPX4, as an important protein of cellular antioxidant system, plays an important protective role in ferroptosis. Surprisingly, the endothelial cell-specific knockout of GPX4 did not damage vascular homeostasis in mice with a normal diet (Wortmann et al., 2013). Similarly, in vitro treatment of vitamin E deficient medium resulted in GPX4 knockout endothelial cell ferroptosis. Under these conditions, when mice were fed a diet deficient in antioxidant vitamin $\mathrm{E}$, thrombotic events occurred in almost all mice. This may indicate that we can avoid ferroptosis and endothelial cell dysfunction by regular intake of antioxidants such as vitamin E. Besides, when the human body inhales PM2.5, the molecular mechanisms of ferroptosis in endothelial cells as the first line of defense include GSH depletion combined with iron metabolism disorder (Wang and Tang, 2019). Zinc oxide nanoparticles (ZnONPs), widely used in cosmetics, rubber, and pigments, promote NCOA4-mediated ferritinophagy in endothelial cells (Qin et al., 2021). Ferroptosis of endothelial cells mediated by ferritinophagy causes endothelial dysfunction and intense vascular inflammation. In summary, endothelial cells ferroptosis plays an important role in promoting atherosclerosis, and inhibition of ferroptosis by improving antioxidant metabolism may be a new target for protecting atherosclerosis.

\section{FERROPTOSIS AND MYOCARDIAL ISCHEMIA REPERFUSION INJURY}

Coronary artery subtotal occlusion or complete thrombosisinduced occlusion is likely to cause the onset of AMI. When AMI occurs, the blood supply to myocardial cells is interrupted and the cells are in a state of severe hypoxia. Unfortunately, the rapid improvement in blood supply in the ischemic myocardium can aggravate the degree of myocardial injury. Ischemiareperfusion is one of the main mechanisms of myocardial injury (IRI) caused by AMI, and can be responsible for up to $50 \%$ of the infracted myocardial area (Lillo-Moya et al., 2021). The previous theory believed that IRI was related to xanthine oxidase and NADPH oxidase levels, and to mitochondrial activity, but new research shows that ferroptosis also plays an important role. (Tang et al., 2021b). found that only during the reperfusion period, the expression of ferroptosis markers MDA 
and ACSL4 was significantly increased, accompanied by a downregulation of GPX4 expression in the Sprague-Dawley rats (SD) model of IRI (Tang et al., 2021a). SD rats injected with the ferroptosis inhibitor DFO before IRI have up-regulated GPX4 expression during reperfusion, accompanied by a decrease in myocardial infarction area and a decrease in creatine kinase (CK) levels. After ischemia-reperfusion, the up-regulation of USP7 expression promotes the progression of ferroptosis through the USP7/P53/TfR1 pathway (Tang et al., 2021b). TfR1 takes transferrin in the blood into cardiomyocytes and provides the initiating factor iron for cardiomyocyte ferroptosis. These studies confirmed that ferroptosis was involved in myocardial IRI. More importantly, ferroptosis of myocardial cells occurs primarily during reperfusion (Tang et al., 2021a).

Diabetes is one of the most important risk factors for AMI. The incidence of myocardial ischemia in patients with diabetes is significantly higher than in the normal population (Ndumele et al., 2016). If diabetic patients experience myocardial IRI, ferroptosis and stress of the endoplasmic reticulum can promote each other and aggravate myocardial IRI within a state of high glucose and hypoxia (Li et al., 2020). In the study, compared to the control group, the degree of myocardial damage in the diabetes group increased dramatically, accompanied by ferroptosis and endoplasmic reticulum stress. Application of the ferroptosis inhibitor Fer-1 or the endoplasmic reticulum stress inhibitor salubrinal or tunicamycin can significantly reduce ferroptosis and endoplasmic reticulum stress, demonstrating that ferroptosis and endoplasmic reticulum stress mutually induce and promote diabetic myocardial IRI.

Existing studies have confirmed the important role of ferroptosis in myocardial ischemia-reperfusion. Therefore, suppressing ferroptosis during ischemia-reperfusion may become a new therapeutic strategy to protect the myocardium. Before reperfusion, Feng et al. administered Lip-1 to mice, a potent specific inhibitor of ferroptosis, which reduced the level of VDAC1 and increased the activity of GPX4 (Feng et al., 2019). Lip-1 also has the ability to maintain mitochondrial function and reduce the area of myocardial infarction. A variety of VDACs are embedded on the surface of the outer mitochondrial membrane (OMM), including VDAC1, VDAC2, and VDAC3. Among them, the oligomerization of VDAC1 induced the occurrence of apoptosis. A mechanism by which erastin induces ferroptosis is that of binding directly to VDAC2 on the surface of the OMM, which changes the permeability of mitochondria (Keinan et al., 2013). Lip-1 induces the opposite effect of erastin and cannot act on VDAC2. Lip-1 shuts down VDAC1 on the surface of OMM and weakens the ability of the VDAC1/GRP75/IP3R1 complex to transport $\mathrm{Ca}^{2+}$ to mitochondria, which inhibits cardiomyocyte apoptosis. Moreover, Lip-1 can strongly eliminate ROS and significantly reduces the ferroptosis of myocardial cells (Keinan et al., 2013). Therefore, Lip-1 may be a drug with great potential to protect cardiomyocytes from IRI.

\section{FERROPTOSIS AND HEART FAILURE}

Subsequent to insufficient blood supply and myocardial IRI caused by coronary atherosclerotic heart disease, a considerable number of patients exhibit a reduction in the number of myocardial cells and present severely damaged cardiac contractile function (Yancy et al., 2013). HF is a highly heterogeneous disease and is the common final result of various cardiovascular diseases, including coronary heart disease, cardiomyopathy, IRI, valvular disease, and hypertension, which develop to the advanced stage (Lakhal-Littleton et al., 2015). In addition to the fact that ferroptosis plays a driving role in various diseases causing HF there are also unique factors that induce ferroptosis to accelerate the progression of HF. In the development of HF, the imbalance of cardiac iron homeostasis can accelerate ferroptosis-mediated HF. Various iron metabolism abnormalities ultimately affect the content of labile iron pool in cells. If the FPN expressed by cardiomyocytes is specifically knocked out, the mice will show a significant increase in left ventricular diameter with a decrease in the ejection fraction (Fang et al., 2020). The non-specific knockout of the Hamp gene, which encodes hepcidin, in mice has been reported to lead to increased iron accumulation in the mouse heart, but did not significantly lead to the onset of cardiovascular disease (Zheng et al., 2021). This is because after hepcidin knockout, although the overall iron content in the heart increased significantly, iron was deposited mainly in noncardiomyocytes, which is the key difference between the two models. Similarly, knocking out Fth in the mouse heart specifically causes intracellular iron metabolism disorders, which can also lead to severe myocardial hypertrophy and myocardial damage (Chen et al., 2019). In addition, the study by Ito et al. found that the mice that specifically knocked out NCOA4 had significantly stronger cardiac function than the wild group after performing transverse aortic constriction (Ito et al., 2021). Their study confirmed that knocking out NCOA4 reduces the occurrence of ferritinophagy, which provides a beneficial help for inhibiting labile iron pool. Other studies confirmed that overexpression of SLC7A11 or exposure to of Fer-1 treatment in mice abolished pathological changes in the myocardium, which showed that myocardial iron metabolism disorder caused heart failure through ferroptosis of myocardial cells (Stevenson et al., 2019).

The noncoding RNA circSnx12 can up-regulate the downstream target miR224-5p, and can combine with the $3^{\prime}$ UTR region of FTH1 to down-regulate its expression (Zheng et al., 2021). In addition to iron metabolism disorders, there are other mechanisms involved in the process of HF. The receptor 4 (TLR4)-NADPH oxidase 4 (NOX4) pathway is up-regulated in cardiomyocytes of mouse models of HF (Chen et al., 2019). TLR4 is the key to activating inflammation, and activation of inflammation promotes the appearance of cardiomyocyte autophagy and the up-regulation of NOX4. NOX4 is the downstream executor of cardiomyocyte ferroptosis. Another study found that NOX4 in heart samples from patients with advanced HF of ischemic cardiomyopathy promoted myocardial inflammation and myocardial fibrosis, which can be mutually verified with the above study (Stevenson et al., 2019). Myocardial fibrosis is the pathological basis for the heart remodeling process in HF. Mixed lineage kinase 3 (MLK3), a member of the MAP3K family, causes myocardial fibrosis by promoting ferroptosis in the late stage of congestive HF (J. Wang et al., 2020). It is worth 
mentioning that the plant antioxidant puerarin is an effective inhibitor of ferroptosis during heart failure. Puerarin can inhibit NOX4 activity and, at the same time, up-regulate the expression of GPX4 and FTH1, and thus, it can delay cardiomyocyte damage and deterioration of heart function (Liu et al., 2018). In the future, including ferroptosis as a therapeutic target may represent an effective breakthrough in the treatment of heart failure.

\section{FERROPTOSIS AND CLINICAL APPLICATION}

With the development of basic research related to ferroptosis, combining ferroptosis with clinical applications has gradually become the next important research direction. The clinical application of ferroptosis-related targets is currently in its infancy. The current clinical research results may enlighten us several potential intervention targets in clinical applications. Since it is difficult to collect ferroptosis indicators in clinical practice, most studies focus on iron metabolity-related indicators, such as serum iron, serum ferritin, transferrin and soluble transferrin receptors. These targets with translational medicine potential could be key to controlling the progression of cardiovascular disease in the future.

Since Sullivan's hypothesis, people have focused on the relationship between serum iron and atherosclerotic diseases (Sullivan, 1981). The incidence of acute myocardial infarction was significantly higher in patients with elevated serum ferritin than in patients with reduced serum ferritin (Moradi et al., 2015). However, lower ferritin levels did not improve all-cause mortality from cardiovascular disease (Zacharski et al., 2007). There are many factors influencing the level of serum ferritin. Under pathological conditions such as inflammation and tumors, the level of serum ferritin will increase significantly. Therefore, we need more accurate predictors to link ferroptosis with coronary atherosclerotic heart disease. The soluble transferrin receptor (sTfR) is derived from the cell membrane surface receptor through hydrolysis, and it exists as a complex with transferrin in the blood. Although the concentration of soluble ferritin receptors is not directly related to the risk of coronary atherosclerotic heart disease, the concentration of sTfR increases with the number of affected coronary arteries (Braun et al., 2004). Hepcidin concentration also has limited predictive value for the risk of future myocardial infarction or cardiovascular death (Zeller et al., 2018). However, intrinsic Iron release, defined as low levels of hepcidin $(<24 \mathrm{ng} / \mathrm{ml})$ and high levels of sTfR $(\geq 2 \mathrm{mg} / \mathrm{L})$, has been associated with a significant reduction in cardiovascular mortality in patients with coronary atherosclerotic heart disease (Ruhe et al., 2018).

Elevated levels of ferritin and hepcidin were highly associated with a higher risk of new-onset heart failure in women but not in men (Klip et al., 2017). Alternatively, disorders in iron intake and absorption in patients with heart failure often result in iron deficiency (ID). Iron not only causes ferroptosis in the body, but also assists in oxygen transport and mitochondrial function. Hence, iron supplementation has become a widely accepted treatment for heart failure patients with ID. Intravenous administration of ferric carboxymaltose for 1 year significantly improves the results of 6-min walking trials in both anemic and non-anemic patients (Ponikowski et al., 2015). In patients with heart failure with ID, serum iron metabolism-related proteins could not correctly predict cardiac ferroptosis levels and heart failure mortality. All in all, it is difficult to use ferroptosis indicators as accurate predictors or therapeutic targets in clinical practice so far.

\section{DISCUSSION}

In recent years, research on the occurrence of ferroptosis and disease has gradually improved. More and more mechanisms related to the regulation of ferroptosis have been discovered, such as the FSP1-CoQ10-NADPH axis and DHODH antioxidant system. But there are still many unsolved mysteries. To date, the major research directions have focused on ferroptosis and tumors. By studying the regulatory pathways that promote tumor cell ferroptosis, we can provide new directions to prolong the survival of tumor patients. In addition, degenerative diseases of the nervous system, liver disease, and kidney disease are also hotspots in ferroptosis research. Due to the high incidence and mortality of cardiovascular diseases, these have gradually become the number one killer of human health. However, our understanding of the pathogenesis and treatment of cardiovascular disease is limited. After the discovery of apoptosis, researchers have confirmed its important role in cardiovascular disease. With the improved understanding of regulatory cell death, other modalities of regulatory cell death, such as necroptosis and pyrolysis, are believed to occur in cardiovascular diseases. Research on ferroptosis and cardiovascular disease is still in its infancy, and most existing studies have confirmed the existence of ferroptosis in cardiovascular disease. As we gradually deepen our understanding of the role of ferroptosis, this mechanism will become a potential breakthrough point in the treatment of cardiovascular disease.

Research on the mechanism of ferroptosis is a prerequisite for clinical treatment and application. The iron hypothesis has been a controversial issue for many years since it was put forward. The recent discovery that iron metabolism disorder is the first step in ferroptosis may be a strategy to quell the controversial issue of iron hypothesis. According to previous theories, the antioxidant system with GPX4 at the core is the only anti-ferroptosis mechanism. The FSP1-CoQ10-NADPH axis and the DHODH antioxidant system suggest that ferroptosis may be regulated by a variety of independent antioxidant mechanisms. The chemical structure of phospholipids is also a target for regulating ferroptosis. The structure of the phospholipid bilayer membrane of different ratios of fatty acid composition gives cells different degrees of vulnerability to ferroptosis. The discovery that conjugated and nonconjugated fatty acids have different ferroptosis characteristics has provided a novel insight for a strategies for intervention, that is, in the future, it is possible to alter the proportion of fatty acids by optimizing dietary intake by the population as a strategy to achieve the goals of prevention and treatment. 
In terms of basic research, ferroptosis has been investigated in terms of coronary atherosclerotic heart disease progression to IRI and even HF. Existing research is limited to in vitro cell-based experiments and animal studies, and there is almost no verification of the pathological development process mentioned above in human samples. Further verification of the role of ferroptosis in human specimens will be the goal of the next stage of research. Arrhythmia-related diseases have so far been the blind spot in ferroptosis studies. In the future, exploring whether ferroptosis is related to arrhythmia may be part of the research prospects.

In clinical application, iron metabolism indicators can only predict the risk of AMI or initial heart failure in a limited manner. Since iron metabolism-related indicators are interfered by many other factors in the human body, more independent predictors of ferroptosis need to be discovered in the future.

In summary, current research has preliminarily shown that ferroptosis is closely related to a variety of cardiovascular diseases. Regulating ferroptosis will be one of the most promising emerging targets to improve the prognosis and the survival rate of cardiovascular diseases.

\section{REFERENCES}

Alim, I., Caulfield, J. T., Chen, Y., Swarup, V., Geschwind, D. H., Ivanova, E., et al. (2019). Selenium Drives a Transcriptional Adaptive Program to Block Ferroptosis and Treat Stroke. Cell 177, 1262-1279. e25. doi:10.1016/ j.cell.2019.03.032

Allahverdian, S., Chaabane, C., Boukais, K., Francis, G. A., and Bochaton-Piallat, M.-L. (2018). Smooth Muscle Cell Fate and Plasticity in Atherosclerosis. Cardiovasc. Res. 114, 540-550. doi:10.1093/cvr/cvy022

Anthonymuthu, T. S., Tyurina, Y. Y., Sun, W.-Y., Mikulska-Ruminska, K., Shrivastava, I. H., Tyurin, V. A., et al. (2021). Resolving the Paradox of Ferroptotic Cell Death: Ferrostatin-1 Binds to 15lox/Pebp1 Complex, Suppresses Generation of Peroxidized Ete-Pe, and Protects against Ferroptosis. Redox Biol. 38, 101744. doi:10.1016/j.redox.2020.101744

Arbustini, E., Dal Bello, B., Morbini, P., Burke, A. P., Bocciarelli, M., Specchia, G., et al. (1999). Plaque Erosion Is a Major Substrate for Coronary Thrombosis in Acute Myocardial Infarction. Heart 82, 269-272. doi:10.1136/hrt.82.3.269

Bai, T., Li, M., Liu, Y., Qiao, Z., and Wang, Z. (2020). Inhibition of Ferroptosis Alleviates Atherosclerosis through Attenuating Lipid Peroxidation and Endothelial Dysfunction in Mouse Aortic Endothelial Cell. Free Radic. Biol. Med. 160, 92-102. doi:10.1016/j.freeradbiomed.2020.07.026

Bao, W.-D., Pang, P., Zhou, X.-T., Hu, F., Xiong, W., Chen, K., et al. (2021). Loss of Ferroportin Induces Memory Impairment by Promoting Ferroptosis in Alzheimer's Disease. Cell Death Differ 28, 1548-1562. doi:10.1038/s41418020-00685-9

Bao, W. D., Zhou, X. T., Zhou, L. T., Wang, F., Yin, X., Lu, Y., et al. (2020). Targeting miR-124/Ferroportin Signaling Ameliorated Neuronal Cell Death through Inhibiting Apoptosis and Ferroptosis in Aged Intracerebral Hemorrhage Murine Model. Aging cell 19, e13235. doi:10.1111/acel.13235

Bennett, M. R., Sinha, S., and Owens, G. K. (2016). Vascular Smooth Muscle Cells in Atherosclerosis. Circ. Res. 118, 692-702. doi:10.1161/circresaha.115.306361

Bersuker, K., Hendricks, J. M., Li, Z., Magtanong, L., Ford, B., Tang, P. H., et al. (2019). The Coq Oxidoreductase Fsp1 Acts Parallel to Gpx4 to Inhibit Ferroptosis. Nature 575, 688-692. doi:10.1038/s41586-019-1705-2

Braun, S., Ndrepepa, G., Beckerath, N. v., Vogt, W., Schömig, A., and Kastrati, A. (2004). Value of Serum Ferritin and Soluble Transferrin Receptor for Prediction of Coronary Artery Disease and its Clinical Presentations. Atherosclerosis 174, 105-110. doi:10.1016/j.atherosclerosis.2004.01.011

Byon, C. H., Javed, A., Dai, Q., Kappes, J. C., Clemens, T. L., Darley-Usmar, V. M., et al. (2008). Oxidative Stress Induces Vascular Calcification through

\section{AUTHOR CONTRIBUTIONS}

YL, XL, and JY: Conceptualization and writing, HJ and BY: Review and editing.

\section{FUNDING}

This research is supported by the National Natural Science Foundation of China (No. 82061130223 and No. 82072031), HMU Marshal Initiative Funding (HMUMIF-21016) and Fund of Key Laboratory of Myocardial Ischemia, Ministry of Education (KF202020/LX).

\section{ACKNOWLEDGMENTS}

We sincerely thank Charlesworth for assistance in language editing and manuscript writing.

Modulation of the Osteogenic Transcription Factor Runx2 by Akt Signaling. J. Biol. Chem. 283, 15319-15327. doi:10.1074/jbc.M800021200

Chen, D., Fan, Z., Rauh, M., Buchfelder, M., Eyupoglu, I. Y., and Savaskan, N. (2017). Atf4 Promotes Angiogenesis and Neuronal Cell Death and Confers Ferroptosis in a Xct-dependent Manner. Oncogene 36, 5593-5608. doi:10.1038/ onc.2017.146

Chen, X., Xu, S., Zhao, C., and Liu, B. (2019). Role of Tlr4/Nadph Oxidase 4 Pathway in Promoting Cell Death through Autophagy and Ferroptosis during Heart Failure. Biochem. biophysical Res. Commun. 516, 37-43. doi:10.1016/ j.bbrc.2019.06.015

Choi, M. E., Price, D. R., Ryter, S. W., and Choi, A. M. K. (2019). Necroptosis: A Crucial Pathogenic Mediator of Human Disease. JCI insight 4. doi:10.1172/ jci.insight. 128834

Chu, B., Kon, N., Chen, D., Li, T., Liu, T., Jiang, L., et al. (2019). Alox12 Is Required for P53-Mediated Tumour Suppression through a Distinct Ferroptosis Pathway. Nat. Cel Biol 21, 579-591. doi:10.1038/s41556-019-0305-6

Crea, F., and Libby, P. (2017). Acute Coronary Syndromes. Circulation 136, 1155-1166. doi:10.1161/circulationaha.117.029870

Dai, J., Xing, L., Jia, H., Zhu, Y., Zhang, S., Hu, S., et al. (2018). In Vivo Predictors of Plaque Erosion in Patients with St-Segment Elevation Myocardial Infarction: A Clinical, Angiographical, and Intravascular Optical Coherence Tomography Study. Eur. Heart J. 39, 2077-2085. doi:10.1093/eurheartj/ehy101

Davies, M. J., and Thomas, A. (1984). Thrombosis and Acute Coronary-Artery Lesions in Sudden Cardiac Ischemic Death. N. Engl. J. Med. 310, 1137-1140. doi:10.1056/nejm198405033101801

Del Re, D. P., Amgalan, D., Linkermann, A., Liu, Q., and Kitsis, R. N. (2019). Fundamental Mechanisms of Regulated Cell Death and Implications for Heart Disease. Physiol. Rev. 99, 1765-1817. doi:10.1152/ physrev.00022.2018

Demetz, E., Tymoszuk, P., Hilbe, R., Volani, C., Haschka, D., Heim, C., et al. (2020). The Haemochromatosis Gene Hfe and Kupffer Cells Control Ldl Cholesterol Homeostasis and Impact on Atherosclerosis Development. Eur. Heart J. 41, 3949-3959. doi:10.1093/eurheartj/ehaa140

Derwall, M., Malhotra, R., Lai, C. S., Beppu, Y., Aikawa, E., Seehra, J. S., et al. (2012). Inhibition of Bone Morphogenetic Protein Signaling Reduces Vascular Calcification and Atherosclerosis. Arterioscler Thromb. Vasc. Biol. 32, 613-622. doi:10.1161/atvbaha.111.242594

Dierge, E., Debock, E., Guilbaud, C., Corbet, C., Mignolet, E., Mignard, L., et al. (2021). Peroxidation of N-3 and N-6 Polyunsaturated Fatty Acids in the Acidic Tumor Environment Leads to Ferroptosis-Mediated Anticancer Effects. Cel Metab. 33, 1701-1715. doi:10.1016/j.cmet.2021.05.016 
Dixon, S. J., Lemberg, K. M., Lamprecht, M. R., Skouta, R., Zaitsev, E. M., Gleason, C. E., et al. (2012). Ferroptosis: An Iron-dependent Form of Nonapoptotic Cell Death. Cell 149, 1060-1072. doi:10.1016/j.cell.2012.03.042

Dixon, S. J., Patel, D. N., Welsch, M., Skouta, R., Lee, E. D., Hayano, M., et al. (2014). Pharmacological Inhibition of Cystine-Glutamate Exchange Induces Endoplasmic Reticulum Stress and Ferroptosis. eLife 3, e02523. doi:10.7554/ eLife. 02523

Doll, S., Freitas, F. P., Shah, R., Aldrovandi, M., da Silva, M. C., Ingold, I., et al. (2019). Fsp1 Is a Glutathione-independent Ferroptosis Suppressor. Nature 575, 693-698. doi:10.1038/s41586-019-1707-0

Doll, S., Proneth, B., Tyurina, Y. Y., Panzilius, E., Kobayashi, S., Ingold, I., et al. (2017). Acsl4 Dictates Ferroptosis Sensitivity by Shaping Cellular Lipid Composition. Nat. Chem. Biol. 13, 91-98. doi:10.1038/nchembio.2239

Dolma, S., Lessnick, S. L., Hahn, W. C., and Stockwell, B. R. (2003). Identification of Genotype-Selective Antitumor Agents Using Synthetic Lethal Chemical Screening in Engineered Human Tumor Cells. Cancer cell 3, 285-296. doi:10.1016/s1535-6108(03)00050-3

Dong, Y., Chen, H., Gao, J., Liu, Y., Li, J., and Wang, J. (2019). Molecular Machinery and Interplay of Apoptosis and Autophagy in Coronary Heart Disease. J. Mol. Cell. Cardiol. 136, 27-41. doi:10.1016/j.yjmcc.2019.09.001

Donovan, A., Lima, C. A., Pinkus, J. L., Pinkus, G. S., Zon, L. I., Robine, S., et al. (2005). The Iron Exporter Ferroportin/Slc40al Is Essential for Iron Homeostasis. Cel Metab. 1, 191-200. doi:10.1016/j.cmet.2005.01.003

Döring, Y., Soehnlein, O., and Weber, C. (2017). Neutrophil Extracellular Traps in Atherosclerosis and Atherothrombosis. Circ. Res. 120, 736-743. doi:10.1161/ circresaha.116.309692

Durham, A. L., Speer, M. Y., Scatena, M., Giachelli, C. M., and Shanahan, C. M. (2018). Role of Smooth Muscle Cells in Vascular Calcification: Implications in Atherosclerosis and Arterial Stiffness. Cardiovasc. Res. 114, 590-600. doi:10.1093/cvr/cvy010

Ezekowitz, J. A., Kaul, P., Bakal, J. A., Armstrong, P. W., Welsh, R. C., and McAlister, F. A. (2009). Declining In-Hospital Mortality and Increasing Heart Failure Incidence in Elderly Patients with First Myocardial Infarction. J. Am. Coll. Cardiol. 53, 13-20. doi:10.1016/j.jacc.2008.08.067

Fang, X., Cai, Z., Wang, H., Han, D., Cheng, Q., Zhang, P., et al. (2020). Loss of Cardiac Ferritin H Facilitates Cardiomyopathy via Slc7a11-Mediated Ferroptosis. Circ. Res. 127, 486-501. doi:10.1161/circresaha.120.316509

Feng, H., Schorpp, K., Jin, J., Yozwiak, C. E., Hoffstrom, B. G., Decker, A. M., et al. (2020). Transferrin Receptor Is a Specific Ferroptosis Marker. Cel Rep. 30, 3411-3423. e7. doi:10.1016/j.celrep.2020.02.049

Feng, Y., Madungwe, N. B., Imam Aliagan, A. D., Tombo, N., and Bopassa, J. C. (2019). Liproxstatin-1 Protects the Mouse Myocardium against Ischemia/ Reperfusion Injury by Decreasing Vdac1 Levels and Restoring Gpx4 Levels. Biochem. biophysical Res. Commun. 520, 606-611. doi:10.1016/ j.bbrc.2019.10.006

Finn, A. V., Nakano, M., Narula, J., Kolodgie, F. D., and Virmani, R. (2010). Concept of Vulnerable/Unstable Plaque. Atvb 30, 1282-1292. doi:10.1161/ atvbaha.108.179739

Friedmann Angeli, J. P., Schneider, M., Proneth, B., Tyurina, Y. Y., Tyurin, V. A., Hammond, V. J., et al. (2014). Inactivation of the Ferroptosis Regulator Gpx4 Triggers Acute Renal Failure in Mice. Nat. Cel Biol 16, 1180-1191. doi:10.1038/ ncb3064

Ganz, T. (2013). Systemic Iron Homeostasis. Physiol. Rev. 93, 1721-1741. doi:10.1152/physrev.00008.2013

Gao, M., Monian, P., Quadri, N., Ramasamy, R., and Jiang, X. (2015). Glutaminolysis and Transferrin Regulate Ferroptosis. Mol. Cel. 59, 298-308. doi:10.1016/j.molcel.2015.06.011

Gaschler, M. M., Andia, A. A., Liu, H., Csuka, J. M., Hurlocker, B., Vaiana, C. A., et al. (2018). FINO2 Initiates Ferroptosis through GPX4 Inactivation and Iron Oxidation. Nat. Chem. Biol. 14, 507-515. doi:10.1038/s41589-0180031-6

Habas, K., and Shang, L. (2018). Alterations in Intercellular Adhesion Molecule 1 (Icam-1) and Vascular Cell Adhesion Molecule 1 (Vcam-1) in Human Endothelial Cells. Tissue and Cell 54, 139-143. doi:10.1016/j.tice.2018.09.002

Habib, E., Linher-Melville, K., Lin, H.-X., and Singh, G. (2015). Expression of XCT and Activity of System Xc- Are Regulated by NRF2 in Human Breast Cancer Cells in Response to Oxidative Stress. Redox Biol. 5, 33-42. doi:10.1016/ j.redox.2015.03.003
Hambright, W. S., Fonseca, R. S., Chen, L., Na, R., and Ran, Q. (2017). Ablation of Ferroptosis Regulator Glutathione Peroxidase 4 in Forebrain Neurons Promotes Cognitive Impairment and Neurodegeneration. Redox Biol. 12, 8-17. doi:10.1016/j.redox.2017.01.021

Hangauer, M. J., Viswanathan, V. S., Ryan, M. J., Bole, D., Eaton, J. K., Matov, A., et al. (2017). Drug-Tolerant Persister Cancer Cells Are Vulnerable to Gpx4 Inhibition. Nature 551, 247-250. doi:10.1038/nature24297

Hong, X., Roh, W., Sullivan, R. J., Wong, K. H. K., Wittner, B. S., Guo, H., et al. (2021). The Lipogenic Regulator Srebp2 Induces Transferrin in Circulating Melanoma Cells and Suppresses Ferroptosis. Cancer Discov. 11, 678-695. doi:10.1158/2159-8290.Cd-19-1500

Hutter, R., Valdiviezo, C., Sauter, B. V., Savontaus, M., Chereshnev, I., Carrick, F. E., et al. (2004). Caspase-3 and Tissue Factor Expression in Lipid-Rich Plaque Macrophages. Circulation 109, 2001-2008. doi:10.1161/ 01.Cir.0000125526.91945

Ingold, I., Berndt, C., Schmitt, S., Doll, S., Poschmann, G., Buday, K., et al. (2018). Selenium Utilization by Gpx4 Is Required to Prevent Hydroperoxide-Induced Ferroptosis. Cell 172, 409-422. e21. doi:10.1016/j.cell.2017.11.048

Ito, J., Omiya, S., Rusu, M.-C., Ueda, H., Murakawa, T., Tanada, Y., et al. (2021). Iron Derived from Autophagy-Mediated Ferritin Degradation Induces Cardiomyocyte Death and Heart Failure in Mice. eLife 10. doi:10.7554/ eLife.62174

Jiang, L., Kon, N., Li, T., Wang, S.-J., Su, T., Hibshoosh, H., et al. (2015). Ferroptosis as a P53-Mediated Activity during Tumour Suppression. Nature 520, 57-62. doi:10.1038/nature14344

Kagan, V. E., Mao, G., Qu, F., Angeli, J. P. F., Doll, S., Croix, C. S., et al. (2017). Oxidized Arachidonic and Adrenic Pes Navigate Cells to Ferroptosis. Nat. Chem. Biol. 13, 81-90. doi:10.1038/nchembio.2238

Karunakaran, D., Geoffrion, M., Wei, L., Gan, W., Richards, L., Shangari, P., et al. (2016). Targeting Macrophage Necroptosis for Therapeutic and Diagnostic Interventions in Atherosclerosis. Sci. Adv. 2, e1600224. doi:10.1126/ sciadv. 1600224

Keinan, N., Pahima, H., Ben-Hail, D., and Shoshan-Barmatz, V. (2013). The Role of Calcium in Vdacl Oligomerization and Mitochondria-Mediated Apoptosis. Biochim. Biophys. Acta (Bba) - Mol. Cel Res. 1833, 1745-1754. doi:10.1016/ j.bbamcr.2013.03.017

Kirii, H., Niwa, T., Yamada, Y., Wada, H., Saito, K., Iwakura, Y., et al. (2003). Lack of Interleukin-1 $\beta$ Decreases the Severity of Atherosclerosis in ApoE-Deficient Mice. Atvb 23, 656-660. doi:10.1161/01.Atv.0000064374.15232.C3

Klip, I. T., Voors, A. A., Swinkels, D. W., Bakker, S. J. L., Kootstra-Ros, J. E., Lam, C. S., et al. (2017). Serum Ferritin and Risk for New-Onset Heart Failure and Cardiovascular Events in the Community. Eur. J. Heart Fail. 19, 348-356. doi:10.1002/ejhf.622

Lakhal-Littleton, S., Wolna, M., Carr, C. A., Miller, J. J. J., Christian, H. C., Ball, V., et al. (2015). Cardiac Ferroportin Regulates Cellular Iron Homeostasis and Is Important for Cardiac Function. Proc. Natl. Acad. Sci. USA 112, 3164-3169. doi:10.1073/pnas.1422373112

Lang, X., Green, M. D., Wang, W., Yu, J., Choi, J. E., Jiang, L., et al. (2019). Radiotherapy and Immunotherapy Promote Tumoral Lipid Oxidation and Ferroptosis via Synergistic Repression of Slc7a11. Cancer Discov. 9, 1673-1685. doi:10.1158/2159-8290.Cd-19-0338

Lewerenz, J., Hewett, S. J., Huang, Y., Lambros, M., Gout, P. W., Kalivas, P. W., et al. (2013). The Cystine/Glutamate Antiporter System Xc- in Health and Disease: From Molecular Mechanisms to Novel Therapeutic Opportunities. Antioxid. Redox Signaling 18, 522-555. doi:10.1089/ars.2011.4391

Li, W., Li, W., Leng, Y., Xiong, Y., and Xia, Z. (2020). Ferroptosis Is Involved in Diabetes Myocardial Ischemia/Reperfusion Injury through Endoplasmic Reticulum Stress. DNA Cel. Biol. 39, 210-225. doi:10.1089/dna.2019.5097

Libby, P., Ridker, P. M., and Hansson, G. K. (2011). Progress and Challenges in Translating the Biology of Atherosclerosis. Nature 473, 317-325. doi:10.1038/ nature10146

Lillo-Moya, J., Rojas-Solé, C., Muñoz-Salamanca, D., Panieri, E., Saso, L., and Rodrigo, R. (2021). Targeting Ferroptosis against Ischemia/Reperfusion Cardiac Injury. Antioxidants 10, 667. doi:10.3390/antiox10050667

Liu, B., Zhao, C., Li, H., Chen, X., Ding, Y., and Xu, S. (2018). Puerarin Protects against Heart Failure Induced by Pressure Overload through Mitigation of Ferroptosis. Biochem. biophysical Res. Commun. 497, 233-240. doi:10.1016/ j.bbrc.2018.02.061 
Lockshin, R. A., and Zakeri, Z. (2001). Programmed Cell Death and Apoptosis: Origins of the Theory. Nat. Rev. Mol. Cel Biol 2, 545-550. doi:10.1038/35080097

Ma, W.-Q., Sun, X.-J., Zhu, Y., and Liu, N.-F. (2021). Metformin Attenuates Hyperlipidaemia-Associated Vascular Calcification through Anti-ferroptotic Effects. Free Radic. Biol. Med. 165, 229-242. doi:10.1016/ j.freeradbiomed.2021.01.033

Magtanong, L., Ko, P. J., and Dixon, S. J. (2016). Emerging Roles for Lipids in Nonapoptotic Cell Death. Cel Death Differ 23, 1099-1109. doi:10.1038/cdd.2016.25

Maguire, E. M., Pearce, S. W. A., and Xiao, Q. (2019). Foam Cell Formation: A New Target for Fighting Atherosclerosis and Cardiovascular Disease. Vasc. Pharmacol. 112, 54-71. doi:10.1016/j.vph.2018.08.002

Mallat, Z., Corbaz, A., Scoazec, A., Graber, P., Alouani, S., Esposito, B., et al. (2001). Interleukin-18/Interleukin-18 Binding Protein Signaling Modulates Atherosclerotic Lesion Development and Stability. Circ. Res. 89, E41-E45. doi:10.1161/hh1901.098735

Mancias, J. D., Wang, X., Gygi, S. P., Harper, J. W., and Kimmelman, A. C. (2014). Quantitative Proteomics Identifies Ncoa4 as the Cargo Receptor Mediating Ferritinophagy. Nature 509, 105-109. doi:10.1038/nature13148

Mao, C., Liu, X., Zhang, Y., Lei, G., Yan, Y., Lee, H., et al. (2021). Dhodh-Mediated Ferroptosis Defence Is a Targetable Vulnerability in Cancer. Nature 593, 586-590. doi:10.1038/s41586-021-03539-7

Mascitelli, L., and Goldstein, M. R. (2014). Hereditary Hemochromatosis, Iron, Hepcidin, and Coronary Heart Disease. Med. hypotheses 82, 402-403. doi:10.1016/j.mehy.2013.12.013

Moradi, M., Fariba, F., and Mohasseli, A. S. (2015). Relation between the Serum Ferritin Level and the Risk for Acute Myocardial Infarction. J. Res. Health Sci. $15,147-151$.

Ndumele, C. E., Matsushita, K., Lazo, M., Bello, N., Blumenthal, R. S., Gerstenblith, G., et al. (2016). Obesity and Subtypes of Incident Cardiovascular Disease. Jaha 5. doi:10.1161/jaha.116.003921

Nemeth, E., Tuttle, M. S., Powelson, J., Vaughn, M. B., Donovan, A., Ward, D. M., et al. (2004). Hepcidin Regulates Cellular Iron Efflux by Binding to Ferroportin and Inducing its Internalization. Science 306, 2090-2093. doi:10.1126/ science. 1104742

Nicoll, R., and Henein, M. Y. (2014). The Predictive Value of Arterial and Valvular Calcification for Mortality and Cardiovascular Events. IJC Heart \& VesselsHeart \& vessels. 3, 1-5. doi:10.1016/j.ijchv.2014.02.001

Park, E., and Chung, S. W. (2019). Ros-Mediated Autophagy Increases Intracellular Iron Levels and Ferroptosis by Ferritin and Transferrin Receptor Regulation. Cell Death Dis 10, 822. doi:10.1038/s41419-019-2064-5

Pfeiler, S., Winkels, H., Kelm, M., and Gerdes, N. (2019). Il-1 Family Cytokines in Cardiovascular Disease. Cytokine 122, 154215. doi:10.1016/j.cyto.2017.11.009

Ponikowski, P., van Veldhuisen, D. J., Comin-Colet, J., Ertl, G., Komajda, M., Mareev, V., et al. (2015). Beneficial Effects of Long-Term Intravenous Iron Therapy with Ferric Carboxymaltose in Patients with Symptomatic Heart Failure and Iron Deficiency. Eur. Heart J. 36, 657-668. doi:10.1093/ eurheartj/ehu385

Qin, X., Zhang, J., Wang, B., Xu, G., Yang, X., Zou, Z., et al. (2021). Ferritinophagy Is Involved in the Zinc Oxide Nanoparticles-Induced Ferroptosis of Vascular Endothelial Cells. Autophagy 17, 4266-4285. doi:10.1080/ 15548627.2021.1911016

Quyyumi, A. A., and Patel, R. S. (20101979). 55. Dallas, Tex, 1092-1094. doi:10.1161/hypertensionaha.109.148957Endothelial Dysfunction and HypertensionHypertension

Ruhe, J., Waldeyer, C., Ojeda, F., Altay, A., Schnabel, R., Schäfer, S., et al. (2018). Intrinsic Iron Release Is Associated with Lower Mortality in Patients with Stable Coronary Artery Disease-First Report on the Prospective Relevance of Intrinsic Iron Release. Biomolecules 8, 72. doi:10.3390/biom8030072

Rui, T., Wang, H., Li, Q., Cheng, Y., Gao, Y., Fang, X., et al. (2021). Deletion of Ferritin $\mathrm{H}$ in Neurons Counteracts the Protective Effect of Melatonin against Traumatic Brain Injury-induced Ferroptosis. J. Pineal Res. 70, e12704. doi:10.1111/jpi.12704

Saeed, O., Otsuka, F., Polavarapu, R., Karmali, V., Weiss, D., Davis, T., et al. (2012). Pharmacological Suppression of Hepcidin Increases Macrophage Cholesterol Efflux and Reduces Foam Cell Formation and Atherosclerosis. Arterioscler Thromb. Vasc. Biol. 32, 299-307. doi:10.1161/atvbaha.111.240101

Sampilvanjil, A., Karasawa, T., Yamada, N., Komada, T., Higashi, T., Baatarjav, C., et al. (2020). Cigarette Smoke Extract Induces Ferroptosis in Vascular Smooth
Muscle Cells. Am. J. Physiology-Heart Circulatory PhysiologyHeart circulatory Physiol. 318, H508-H518. doi:10.1152/ajpheart.00559.2019

Seta, Y., Kanda, T., Tanaka, T., Arai, M., Sekiguchi, K., Yokoyama, T., et al. (2000). Interleukin 18 in Acute Myocardial Infarction. Heart (British Cardiac Society) 84, 668-669. doi:10.1136/heart.84.6.668

Song, X., Zhu, S., Chen, P., Hou, W., Wen, Q., Liu, J., et al. (2018). AMPK-mediated BECN1 Phosphorylation Promotes Ferroptosis by Directly Blocking System Xc- Activity. Curr. Biol. 28, 2388-2399. e5. doi:10.1016/j.cub.2018.05.094

Stevenson, M. D., Canugovi, C., Vendrov, A. E., Hayami, T., Bowles, D. E., Krause, K.-H., et al. (2019). Nadph Oxidase 4 Regulates Inflammation in Ischemic Heart Failure: Role of Soluble Epoxide Hydrolase. Antioxid. Redox Signaling 31, 39-58. doi:10.1089/ars.2018.7548

Su, G., Yang, W., Wang, S., Geng, C., and Guan, X. (2021). SIRT1-autophagy axis Inhibits Excess Iron-Induced Ferroptosis of Foam Cells and Subsequently Increases IL-1B and IL-18. Biochem. biophysical Res. Commun. 561, 33-39. doi:10.1016/j.bbrc.2021.05.011

Sullivan, J. (1981). Iron and the Sex Difference in Heart Disease Risk. The Lancet 317, 1293-1294. doi:10.1016/s0140-6736(81)92463-6

Sun, X., Ou, Z., Xie, M., Kang, R., Fan, Y., Niu, X., et al. (2015). Hspb1 as a Novel Regulator of Ferroptotic Cancer Cell Death. Oncogene 34, 5617-5625. doi:10.1038/onc.2015.32

Tabas, I. (2010). Macrophage Death and Defective Inflammation Resolution in Atherosclerosis. Nat. Rev. Immunol. 10, 36-46. doi:10.1038/nri2675

Tang, L.-J., Luo, X.-J., Tu, H., Chen, H., Xiong, X.-M., Li, N.-S., et al. (2021a). Ferroptosis Occurs in Phase of Reperfusion but Not Ischemia in Rat Heart Following Ischemia or Ischemia/Reperfusion. Naunyn-schmiedeberg's Arch. Pharmacol. 394, 401-410. doi:10.1007/s00210-020-01932-Z

Tang, L.-J., Zhou, Y.-J., Xiong, X.-M., Li, N.-S., Zhang, J.-J., Luo, X.-J., et al. (2021b). Ubiquitin-Specific Protease 7 Promotes Ferroptosis via Activation of the P53/ Tfr1 Pathway in the Rat Hearts after Ischemia/Reperfusion. Free Radic. Biol. Med. 162, 339-352. doi:10.1016/j.freeradbiomed.2020.10.307

Trpkovic, A., Resanovic, I., Stanimirovic, J., Radak, D., Mousa, S. A., CenicMilosevic, D., et al. (2015). Oxidized Low-Density Lipoprotein as a Biomarker of Cardiovascular Diseases. Crit. Rev. Clin. Lab. Sci. 52, 70-85. doi:10.3109/ 10408363.2014.992063

Venkatesh, D., O'Brien, N. A., Zandkarimi, F., Tong, D. R., Stokes, M. E., Dunn, D. E., et al. (2020). MDM2 and MDMX Promote Ferroptosis by PPARa-Mediated Lipid Remodeling. Genes Dev. 34, 526-543. doi:10.1101/gad.334219.119

Verhoye, E., and Langlois, M. R. (2009). Circulating Oxidized Low-Density Lipoprotein: A Biomarker of Atherosclerosis and Cardiovascular Risk? Clin. Chem. Lab. Med. 47, 128-137. doi:10.1515/cclm.2009.037

Viswanathan, V. S., Ryan, M. J., Dhruv, H. D., Gill, S., Eichhoff, O. M., SeashoreLudlow, B., et al. (2017). Dependency of a Therapy-Resistant State of Cancer Cells on a Lipid Peroxidase Pathway. Nature 547, 453-457. doi:10.1038/ nature23007

Wang, D., Yang, Y., Lei, Y., Tzvetkov, N. T., Liu, X., Yeung, A. W. K., et al. (2019). Targeting Foam Cell Formation in Atherosclerosis: Therapeutic Potential of Natural Products. Pharmacol. Rev. 71, 596-670. doi:10.1124/ pr.118.017178

Wang, J., Deng, B., Liu, Q., Huang, Y., Chen, W., Li, J., et al. (2020). Pyroptosis and Ferroptosis Induced by Mixed Lineage Kinase 3 (Mlk3) Signaling in Cardiomyocytes Are Essential for Myocardial Fibrosis in Response to Pressure Overload. Cel Death Dis 11, 574. doi:10.1038/ s41419-020-02777-3

Wang, K., Zhang, Z., Tsai, H.-i., Liu, Y., Gao, J., Wang, M., et al. (2021). BranchedChain Amino Acid Aminotransferase 2 Regulates Ferroptotic Cell Death in Cancer Cells. Cel Death Differ 28, 1222-1236. doi:10.1038/s41418-020-00644-4

Wang, L., Liu, Y., Du, T., Yang, H., Lei, L., Guo, M., et al. (2020). ATF3 Promotes Erastin-Induced Ferroptosis by Suppressing System Xc-. Cel Death Differ 27, 662-675. doi:10.1038/s41418-019-0380-z

Wang, W., Green, M., Choi, J. E., Gijón, M., Kennedy, P. D., Johnson, J. K., et al. (2019). CD8+ T Cells Regulate Tumour Ferroptosis during Cancer Immunotherapy. Nature 569, 270-274. doi:10.1038/s41586-019-1170-y

Wang, Y., and Tang, M. (2019). Pm2.5 Induces Ferroptosis in Human Endothelial Cells through Iron Overload and Redox Imbalance. Environ. Pollut. 254, 112937. doi:10.1016/j.envpol.2019.07.105

Weissberg, P. L., and Bennett, M. R. (1999). Atherosclerosis - an Inflammatory Disease. N. Engl. J. Med. 340, 1928-1929. doi:10.1056/nejm199906173402418 
Wenzel, S. E., Tyurina, Y. Y., Zhao, J., St. Croix, C. M., Dar, H. H., Mao, G., et al. (2017). Pebp1 Wardens Ferroptosis by Enabling Lipoxygenase Generation of Lipid Death Signals. Cell 171, 628-641. e26. doi:10.1016/j.cell.2017.09.044

Wiktorowska-Owczarek, A., Berezińska, M., and Nowak, J. (2015). Pufas: Structures, Metabolism and Functions. Adv. Clin. Exp. Med. 24, 931-941. doi: $10.17219 /$ acem $/ 31243$

Wortmann, M., Schneider, M., Pircher, J., Hellfritsch, J., Aichler, M., Vegi, N., et al. (2013). Combined Deficiency in Glutathione Peroxidase 4 and Vitamin E Causes Multiorgan Thrombus Formation and Early Death in Mice. Circ. Res. 113, 408-417. doi:10.1161/circresaha.113.279984

Wunderer, F., Traeger, L., Sigurslid, H. H., Meybohm, P., Bloch, D. B., and Malhotra, R. (2020). The Role of Hepcidin and Iron Homeostasis in Atherosclerosis. Pharmacol. Res. 153, 104664. doi:10.1016/ j.phrs.2020.104664

Yancy, C. W., Jessup, M., Bozkurt, B., Butler, J., Casey, D. E., Drazner, M. H., et al. (2013). 2013 ACCF/AHA Guideline for the Management of Heart Failure. J. Am. Coll. Cardiol. 62, e147-e239. doi:10.1016/j.jacc.2013.05.019

Yang, W. S., SriRamaratnam, R., Welsch, M. E., Shimada, K., Skouta, R., Viswanathan, V. S., et al. (2014). Regulation of Ferroptotic Cancer Cell Death by Gpx4. Cell 156, 317-331. doi:10.1016/j.cell.2013.12.010

Yu, Y., Jiang, L., Wang, H., Shen, Z., Cheng, Q., Zhang, P., et al. (2020). Hepatic Transferrin Plays a Role in Systemic Iron Homeostasis and Liver Ferroptosis. Blood 136, 726-739. doi:10.1182/blood.2019002907

Yuan, H., Li, X., Zhang, X., Kang, R., and Tang, D. (2016). Identification of Acsl4 as a Biomarker and Contributor of Ferroptosis. Biochem. biophysical Res. Commun. 478, 1338-1343. doi:10.1016/j.bbrc.2016.08.124

Zacharski, L. R., Chow, B. K., Howes, P. S., Shamayeva, G., Baron, J. A., Dalman, R. L., et al. (2007). Reduction of Iron Stores and Cardiovascular Outcomes in Patients with Peripheral Arterial Disease. JAMA 297, 603-610. doi:10.1001/ jama.297.6.603

Zeller, T., Altay, A., Waldeyer, C., Appelbaum, S., Ojeda, F., Ruhe, J., et al. (2018). Prognostic Value of Iron-Homeostasis Regulating Peptide Hepcidin in
Coronary Heart Disease-Evidence from the Large Atherogene Study. Biomolecules 8, 43. doi:10.3390/biom8030043

Zhang, Y., Swanda, R. V., Nie, L., Liu, X., Wang, C., Lee, H., et al. (2021). Mtorc1 Couples Cyst(E)Ine Availability with Gpx4 Protein Synthesis and Ferroptosis Regulation. Nat. Commun. 12, 1589. doi:10.1038/s41467-021-21841-w

Zhaolin, Z., Guohua, L., Shiyuan, W., and Zuo, W. (2019). Role of Pyroptosis in Cardiovascular Disease. Cell Prolif 52, e12563. doi:10.1111/cpr.12563

Zheng, H., Shi, L., Tong, C., Liu, Y., and Hou, M. (2021). Circsnx12 Is Involved in Ferroptosis during Heart Failure by Targeting Mir-224-5p. Front. Cardiovasc. Med. 8, 656093. doi:10.3389/fcrm.2021.656093

Zhou, Y., Zhou, H., Hua, L., Hou, C., Jia, Q., Chen, J., et al. (2021). Verification of Ferroptosis and Pyroptosis and Identification of Ptgs2 as the Hub Gene in Human Coronary Artery Atherosclerosis. Free Radic. Biol. Med. 171, 55-68. doi:10.1016/j.freeradbiomed.2021.05.009

Conflict of Interest: The authors declare that the research was conducted in the absence of any commercial or financial relationships that could be construed as a potential conflict of interest.

Publisher's Note: All claims expressed in this article are solely those of the authors and do not necessarily represent those of their affiliated organizations, or those of the publisher, the editors and the reviewers. Any product that may be evaluated in this article, or claim that may be made by its manufacturer, is not guaranteed or endorsed by the publisher.

Copyright (c) 2022 Leng, Luo, Yu, Jia and Yu. This is an open-access article distributed under the terms of the Creative Commons Attribution License (CC $B Y)$. The use, distribution or reproduction in other forums is permitted, provided the original author(s) and the copyright owner(s) are credited and that the original publication in this journal is cited, in accordance with accepted academic practice. No use, distribution or reproduction is permitted which does not comply with these terms. 